\title{
Modeling of Nonlinear Flexible Aircraft Dynamics Including Free-Wake Effects
}

\author{
Joseba Murua ${ }^{1}$, Rafael Palacios ${ }^{2}$ and J. Michael R. Graham ${ }^{3}$ \\ Imperial College London, SW7 2AZ, London, United Kingdom
}

\begin{abstract}
This paper addresses the unified aeroelastic and flight dynamics characterization of lowspeed slender-wing aircraft, including free-wake effects and aerodynamic interference. An analysis framework is presented that targets the prediction of stability and handling qualities of high-altitude long-endurance vehicles, which are prone to experience large wing excursions, leading to an inherently nonlinear and coupled problem between aerodynamics, elasticity and flight dynamics. In this work, the structural dynamics are based on a geometrically-exact composite beam model, discretized using displacement-based finite elements, and cast into an extended flexible-body dynamics model. The aerodynamic model is defined by a general unsteady vortex lattice method. The governing equations of motion of the integrated system are formulated in a tightly-coupled state-space form, which allows for the equations to be solved simultaneously. Verification of the model has been carried out for static and dynamic problems, including both rigid and flexible wings. Numerical studies are presented for the particular case of prescribed rigid-body motions, paying special attention to the likely interference between wake and tail. Results show that the current approach represents a suitable alternative for configuration analysis of flexible atmospheric vehicles, offering a good balance between degree of fidelity and computational cost.
\end{abstract}

\begin{tabular}{|c|c|c|c|}
\hline \multicolumn{4}{|c|}{ Nomenclature } \\
\hline$a$ & $=$ body-attached (global) reference frame & $\Delta p$ & $=$ pressure jump across aerodynamic panel \\
\hline$a_{k l}, a_{k l}^{*}$ & $=$ aerodynamic influence coefficients & $\vec{R}$ & $=$ beam local position vector \\
\hline$B$ & $=$ deformed (local) reference frame & $S$ & $=$ arc length along reference line \\
\hline$b$ & $=$ undeformed (local) reference frame & $t$ & $=$ physical time \\
\hline$\Delta b$ & $=$ spanwise dimension of aerodynamic panel & $V$ & $=$ beam local translational velocity \\
\hline$C^{B a}$ & $\begin{array}{l}=\text { coordinate transformation matrix from } \\
\quad a \text { to } B\end{array}$ & $V_{\infty}$ & $=$ free-stream velocity \\
\hline$\Delta c$ & $=$ chordwise dimension of aerodynamic panel & $\vec{v}$ & $=$ translational velocity of the $a$ frame \\
\hline$G$ & $=$ inertial (ground) frame of reference & $\vec{v}_{k}$ & $=$ downwash \\
\hline$K_{b}, K_{w}$ & $\begin{aligned}= & \text { number of bound and wake vortex-rings } \\
& \text { considered }\end{aligned}$ & $w$ & $\begin{array}{l}=\text { normal component of the non-vortical } \\
\text { induced velocity }\end{array}$ \\
\hline$K, k$ & $=$ current and initial beam local curvatures & $\mathbf{X}$ & $=$ coordinates on aerodynamic lattice \\
\hline$\vec{p}$ & $=$ position of the $a$ frame with respect to $G$ & $x$ & $=$ state vector \\
\hline \multicolumn{4}{|c|}{ Greek letters } \\
\hline$\Gamma$ & $=$ circulation strength & $\vec{\Omega}$ & $=$ beam local angular velocity \\
\hline$\gamma$ & $=$ beam local force strain & $\vec{\omega}$ & $=$ angular velocity of the $a$ frame \\
\hline$\zeta$ & $=$ quaternions & $\Psi$ & $=$ Cartesian Rotation Vector \\
\hline$\kappa$ & $=$ beam local moment strain & & \\
\hline
\end{tabular}

${ }^{1}$ Graduate Student, Department of Aeronautics, AIAA Student Member.

${ }^{2}$ Lecturer, Department of Aeronautics, e-mail: rpalacio@imperial.ac.uk, AIAA Member.

${ }^{3}$ Professor, Department of Aeronautics, AIAA Member. 


\begin{tabular}{|c|c|c|c|}
\hline \multicolumn{4}{|c|}{ Subscripts } \\
\hline$A$ & $=$ aerodynamic & $R$ & $=$ rigid-body \\
\hline$b$ & $=$ bound, corresponding to lifting surface & $S$ & $=$ structural \\
\hline$i, j, k$ & $=$ chordwise, spanwise and total panel counters & $w$ & $=$ wake \\
\hline \multicolumn{4}{|c|}{ Superscripts } \\
\hline$\bullet^{*}$ & $=$ corresponding to wake & $\tilde{\bullet}$ & $=$ cross-product operator \\
\hline$\dot{\bullet}$ & $\begin{array}{l}=\text { derivatives with time, } t \\
=\text { derivatives with the curvilinear coordinate, } s\end{array}$ & $\vec{\bullet}$ & $=$ vector magnitude \\
\hline
\end{tabular}

\section{Introduction}

$\mathrm{H}$ IGH-Altitude Long-Endurance (HALE) vehicles are being developed for multiple applications, including environmental sensing, telecom relay, and military reconnaissance ${ }^{1}$. Although this class of aerial platforms has gained considerable attention over the last few years, available analysis techniques for extreme-endurance unconventional vehicles are yet far from maturity - as exemplified by the unfortunate mishap of the Helios solar aircraft $^{2}$. This project addresses the problem of the accurate performance prediction of Very Flexible Aircraft (VFA), with main focus on the development of computational tools to capture all the relevant physics in the flight dynamics of solar-powered atmospheric vehicles. In particular, this paper will concentrate on the ability to capture possible wake interferences between wing and tail on VFA and their potential effect on flight performance.

Traditional aircraft design methodologies are mainly based on linear models, since for conventional relatively stiff structures, consideration of nonlinear phenomena can be eliminated from the design process. However, solarpowered and similar vehicles are constrained by the available power at their disposal, limited by the efficiency of photovoltaic cells. In order to counteract this effect, these vehicles need to satisfy two main conditions: an extremely light, and hence flexible, structure to minimize power requirements, and a long and slender high-aspect-ratio wing which maximizes lift-to-drag ratio. The wing flexibility coupled with the long span is likely to lead to large deflections during normal flight operation, exhibiting geometrically nonlinear behavior. Large static deflections may change the natural frequencies of the wing, which, in turn, can produce drastic changes in its aeroelastic behavior ${ }^{3}$. For instance, HALE vehicles could experience subcritical bifurcations even below the nominal flutter velocity ${ }^{4}$. However, whether the nonlinear effects are favorable or not will greatly depend on the particular circumstances and physical parameters involved ${ }^{5}$, and therefore, only fully nonlinear aeroelastic tools will provide accurate predictions.

A second key factor that further complicates the prediction of VFA behavior is that it represents an inherently multi-disciplinary problem; the interaction between an extremely lightweight airframe, its aerodynamic characteristics, and the vehicle's flight mechanics must be fully integrated. Traditionally, flight dynamics and aeroelasticity have been analyzed separately, relying on a decoupling of their frequency responses. However, very flexible vehicles will present very low first natural frequencies, and this decoupling is no longer possible. For instance, the first bending mode is likely to couple with the short-period oscillation, giving rise to Body-FreedomFlutter ${ }^{6}$. In addition, when the nonlinear flexibility effects are taken into account in the calculation of trim and flight dynamics characteristics, the predicted aeroelastic behavior of the complete aircraft turns out to be very different from what it would be without such effects ${ }^{5-9}$. A substantial amount of work has been carried out exploring the interaction between aeroelasticity and control systems ${ }^{10}$, but few attempts have been made to include a tight link with the aircraft dynamics.

As a result, it would be desirable to develop accurate computational models tailored to these applications, properly accounting for the dominant linear and nonlinear effects in a fully coupled formulation. For this purpose, classical rigid-body dynamics must be expanded in order to incorporate the flexibility of the aircraft in the analysis, and the above needs to be combined with the aerodynamics in a single framework.

The pioneering work investigating geometrically nonlinear effects on VFA was carried out in the development of the Daedalus human-powered aircraft ${ }^{11}$. This study demonstrated that the separation between rigid-body and flexible modes cannot be justified in this case, and it illustrated the impact of the coupled effects between unsteady aerodynamics, large deflections and flight dynamics. Since then, several groups have pursued further the problem of accurate prediction of flexible vehicle behavior ${ }^{3-9,12-17}$. Although alternative approaches have been explored, the usual way of addressing the problem is to seek a unified formulation by coupling a geometrically-exact beam structural model with an unsteady 2-D aerodynamic model for the wing airfoils and the nonlinear rigid-body equations of motion ${ }^{1,4,9}$. This allows capturing the basic physical phenomena with a relatively small number of 
degrees of freedom and has provided significant evidence of the large effect that structural deformations may have on the aircraft dynamics ${ }^{18}$.

The characteristic slenderness of the different elements that compose a flexible aircraft (wing, fuselage, tail) motivates a one-dimensional beam-like structural modeling. This 1-D beam will undergo three-dimensional displacements and rotations, but its cross-sectional properties can be calculated along the span. In the displacementbased approach presented in Refs. 19 and 20, the secondary beam variables are linearly related to the primary variables by the cross-sectional constitutive laws (flexibility and inertia matrices) and these relations are derived based on the assumptions of small strain and slenderness. In some recent works, the strain of the beam elements ${ }^{6,22,23}$ or the velocities and strains (hybrid formulation ${ }^{24}$ ) have been taken as primary variables. Ref. 18 presents a comparative study on these different beam-based structural models, and the corresponding solution procedures, all based on finite-element discretization. In that work, numerical efficiency and the simplicity for integration in full aircraft flexibility and flight dynamics formulations were explored.

Flexible HALE aerodynamics are commonly modeled using 2-D airfoil models, which leads to a state-space formulation $^{25,26}$ suitable for assembly in the full integrated model. However, this approximation is unable to capture three-dimensional effects, and different corrections have been introduced in order to account for them. A full 3-D aerodynamic model should thus offer a higher fidelity, but it could jeopardize the simplicity of the unified model. Potential-flow-based methods offer a practical alternative to full Navier-Stokes solvers; as long as the potential flow assumption holds (inviscid, incompressible, irrotational), they provide a significant improvement of accuracy with respect to 2-D models, hence rendering them excellent candidates for studies of low-speed flight and attached flow $^{16,27}$. HALE vehicles are likely to fulfill these conditions, at least in a wide range of their design envelope potential flow aerodynamic models might not be adequate at very high altitudes (dominance of viscous effects or breakdown of continuum hypothesis), but this is not relevant for the mission segments of interest (climb and descend), when critical gust encounters and the most demanding maneuvers would take place. If the large wing excursions reach the aerodynamic nonlinear regime (separation), ad hoc corrections might be applied to account for static and dynamic stall ${ }^{28,29}$. A further limitation of potential flow solvers is that they cannot predict viscous drag, which is essential for the flight dynamics analysis. This can be however overcome by computing it using an alternative approach, such as a 2 -D viscous airfoil solver ${ }^{30}$.

Different potential flow models exist where the wake structure can either be prescribed or computed as part of the overall solution procedure. Despite being computationally more expensive, free-wake methods become necessary for unsteady flow situations with complicated wing kinematics. Furthermore, they are indispensable in the investigation of interference problems, such as when the wake encounters a second lifting surface, which is likely to happen in HALE vehicles between wing and tail. Vortices incident upon bodies can give rise to substantial unsteady loading $^{31}$, and the aerodynamic loads of a trailing wing can be affected by the wake of the leading wing even at a 10 chord distance ${ }^{32}$. Note finally that linearized methods in which a flat wake is assumed, such as the Doublet-Lattice Method $^{33,34}$, are not suitable for these problems.

The situation in which the wake of a leading wing crosses a trailing surface is very similar to when a trailing tipvortex shed from one helicopter blade interacts with itself or other blades. Blade-Vortex Interaction (BVI) has been widely investigated in the rotorcraft community ${ }^{35-39}$. The same physics are also observed when wake-dynamics are studied in order to determine safe take-off and landing distances, since the wake of a leading aircraft may affect a following one. This has been investigated using the UVLM by, for instance, by Karkehabadi ${ }^{32}$, whom reported an analogous qualitative behavior to a BVI.

Among free-wake methods, different alternatives exist. On the one hand, vortex-particle methods ${ }^{40,41}$ adopt a "vortical" interpretation of the wake, which consists of point-vortices or vortex-blobs. On the other hand, the general Unsteady Vortex Lattice Method (UVLM) ${ }^{42}$ represents the wake by a continuous vortex-sheet. According to Ref. 43, the UVLM is shown to be a good candidate for, at least, preliminary studies of strongly interfering flowfields and subsequent simulations of the flying qualities of configurations featuring such unsteady interference.

The UVLM obtains the real shape of the wake by using discrete vortex filaments arranged in quadrilateral vortex rings to account for the vorticity in the wake. Discrete vortex filaments originate numerical difficulties due to the singularity of the Biot-Savart law and this will occur in interference situations, when wake filaments cross trailing surfaces. As a result, regularization is required, which can be accomplished in a variety of ways, such as remeshing ${ }^{40}$ or discarding wake connectivity ${ }^{41,44}$, for instance. However, the simplest remedy is to introduce a vortex-core to model the inner viscous part of the vortex filament. Several models have been proposed ${ }^{35,39,45}$, but in all of them the choice of core size lacks theoretical or physical justification. As a consequence, it has to be defined cautiously, since it can have a significant influence on the solution.

The ultimate goal of this work is to present a complete aeroelastic model for a highly flexible aircraft, including wake and interference description. For this purpose, a flexible-body dynamics formulation is presented first, where a 
displacement-based beam structural model ${ }^{19,20}$ has been used (Section II). Secondly, the aerodynamic vortex lattice method $^{42}$ is introduced, which computes the shape of the wake as part of the solution (Section III). Next, the full set of governing equations is cast into an integrated model, formulated in a tightly-coupled discrete state-space fashion, where the nonlinear equations can be solved simultaneously using different integration schemes (Section IV). This framework encompasses rigid-body motions as degrees of freedom. However, for the numerical studies only prescribed aircraft-dynamics are considered and, hence, the simplified equations are presented (Section V). Finally, representative results are included, comparing the described model to published literature for nonlinear problems, and assessing the impact of interference phenomena on the aircraft handling capabilities (Section VI).

\section{Flexible-body dynamics: displacement-based geometrically-exact beam element}

All primary structures of the highly flexible HALE aircraft are modeled via geometrically-nonlinear composite beams, using the displacement-based approach based on the work of Géradin and Cardona ${ }^{20}$ and Simo and VuQuoq $^{21}$, even though the notation mostly follows that of Hodges ${ }^{19}$. First of all, the kinematics of a beam-like slender structure need to be defined, possibly subject to large deflections and global rotations, but under the assumption of small strains and local rotations. These conditions are satisfied by flexible structures experiencing large excursions, but operating in the elastic linear regime.

Under the assumption of slenderness, the 3-D displacement field of the body can be referred to a 1-D geometrically-exact description of the deformations of a reference line, along its longitudinal (dominant) direction. The longitudinal rate of change of the reference line displacements and rotations are then characterized by a set of beam strain measures, while their variations in time define the beam local velocities. A process of structural homogenization from the 3-D geometry to the 1-D model is also required ${ }^{46}$, but this will not be discussed here.

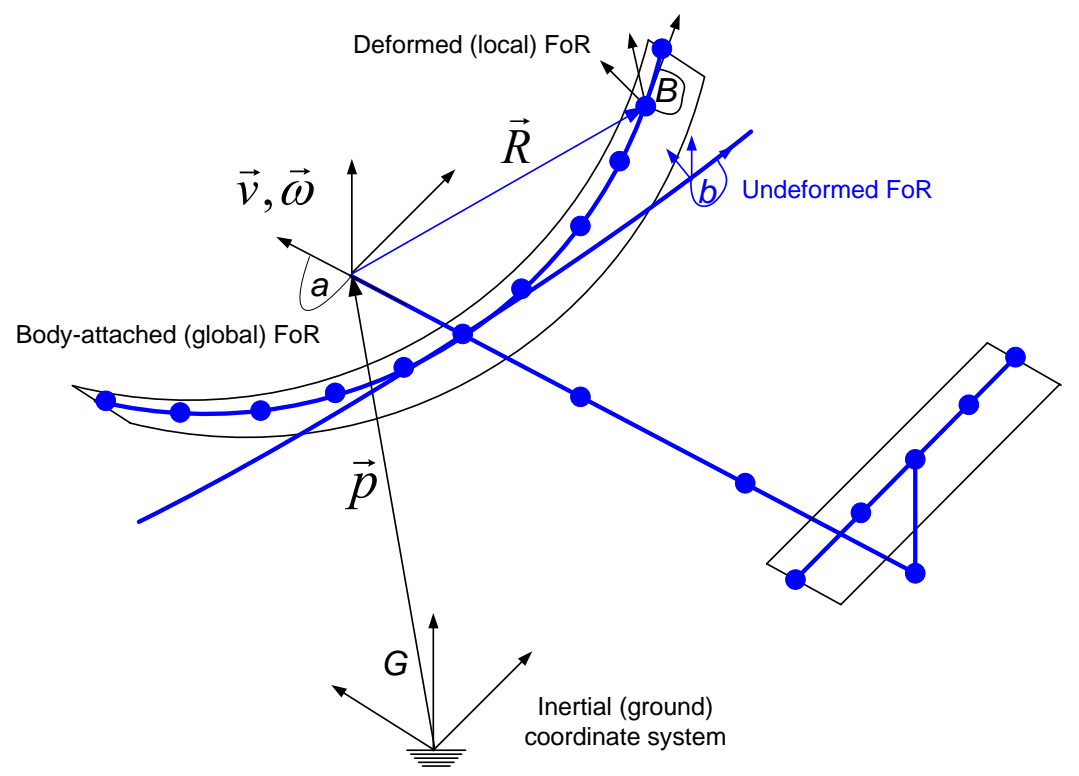

Figure 1. Beam-like discretization of flexible aircraft: undeformed/deformed reference lines and relevant reference frames.

The following reference frames are introduced (see Figure 1): body-attached (global) reference coordinate system, $a$, which is allowed to move in space with both translational, $\vec{v}$, and rotational, $\vec{\omega}$, velocities with respect to an inertial (ground) coordinate system, $G$; local or material coordinate system, $B$, which defines the local orientations along each deformed member in the current configuration; and $b$, the undeformed frame. At $t=0$, the deformed local reference frame, $B$, coincides with the undeformed one, $b$.

Based on Hamilton's principle, the equations of motion of a curved nonlinear beam of arc-length $l$ in the time interval $\left[t_{1}, t_{2}\right]$ can be written, in weak form, as 


$$
\int_{t_{1}}^{t_{2}} \delta \Pi d t=\int_{t_{1}}^{t_{2}} \int_{0}^{l}[\delta T-\delta U+\delta W] d s d t=0
$$

where $\Pi$ is the total potential of the beam, $T$ and $U$ are, respectively, the kinetic and internal energy densities per unit length, $W$ corresponds to the virtual work of the applied loads, per unit length, and the longitudinal coordinate along the beam reference line is given by $S$. Both the kinetic energy and the virtual work will in turn comprise contributions associated to the rigid-body dynamics, on the one hand, and to the flexibility of the beam on the other. Assuming linear constitutive relations and an arbitrary distribution of the anisotropic (composite) material, the energy densities are ${ }^{46}$

$$
\begin{aligned}
& T=\frac{1}{2}\left\{\begin{array}{ll}
V_{B}^{T} & \left.\Omega_{B}^{T}\right\}[M]
\end{array}\right]\left\{\begin{array}{c}
V_{B} \\
\Omega_{B}
\end{array}\right\}, \\
& U=\frac{1}{2}\left\{\begin{array}{ll}
\gamma^{\mathrm{T}} & \left.\kappa^{\mathrm{T}}\right\}[S]\left\{\begin{array}{l}
\gamma \\
\kappa
\end{array}\right\}
\end{array},\right.
\end{aligned}
$$

where $V$ and $\Omega$ are the translational and angular inertial velocities, respectively and $\gamma$ and $\kappa$ correspond to force and moment strains. Sub-indexes are used to indicate the coordinate system in which each vector magnitude is projected. Note that when a vector is expressed as its components on a particular reference frame, the vector notation, $\vec{\bullet}$, is dropped.

Equation (1) is solved together with the nonlinear velocity-displacement and the strain-displacement kinematic relations ${ }^{19}$,

$$
\begin{aligned}
& V_{B}=C^{B a}\left(\dot{R}_{a}+\tilde{\omega}_{a} R_{a}+v_{a}\right) \\
& \tilde{\Omega}_{B}=C^{B a} \dot{C}^{a B}+C^{B a} \tilde{\omega}_{a} C^{a B}
\end{aligned} \quad \text { and } \quad \begin{gathered}
\gamma=C^{B a} R_{a}^{\prime}-\vec{e}_{1}, \\
\tilde{\kappa}=\tilde{K}_{B}-\tilde{k}_{b}
\end{gathered}
$$

where $\tilde{K}_{B}=C^{B a} C^{a B^{\prime}}$ and $\tilde{k}_{b}$ are the current and the initial curvatures, respectively; $C^{B a}$ stands for the coordinate transformation matrix between reference frames, from $a$ to $B$; $\tilde{\boldsymbol{0}}$ represents the skew-symmetric equivalent representation of a vector; $\vec{R}$ corresponds to the local position vector; and $\vec{e}_{1}=\left[\begin{array}{lll}1 & 0 & 0\end{array}\right]^{T}$. The description of the beam kinematics described so far is done independently of any discretization used to solve its equations of motion and several solution procedures ${ }^{18}$ can be devised to solve the problem defined by Eqs. (1)-(3). Here, a finite-element based discretization will be used and the set of independent degrees of freedom will be given by displacements and rotations. For the description of the rotations different parameters can be used, such as Euler or Bryant angles, Rodrigues parameters, quaternions etc. In this work, the Cartesian Rotation $V^{2} \operatorname{ctor}^{20}, \Psi$, has been used, which defines the rotation from the $a$ reference system to the local deformed frame, $B$. The Cartesian rotation vector introduces the minimum number of states (three) without introducing singularities ${ }^{18}$.

The equations governing the flexible-body dynamics and the corresponding initial and boundary conditions, obtained from Eqs. (1)-(3), define a closed set on the degrees of freedom

$$
\beta=\left\{\begin{array}{c}
v_{a} \\
\omega_{a}
\end{array}\right\} \quad \text { and } \quad \eta=\left\{\begin{array}{c}
R_{a} \\
\Psi
\end{array}\right\} .
$$

The beam degrees of freedom, $\eta$, are approximated by a finite-element discretization, i.e., $\eta=N \bar{\eta}$, where $\bar{\eta}$ is the discrete set of degrees of freedom and $N$ the corresponding shape functions. In this work a 2-noded beam element has been implemented, using a single-point Gaussian integration scheme. With these definitions, Eqs. (1)(3) can be expressed in classical mechanics form $\mathrm{as}^{20,21}$

$$
M_{\eta}(\bar{\eta})\left\{\begin{array}{c}
\dot{\beta} \\
\ddot{\bar{\eta}}
\end{array}\right\}+F_{\text {gyr }}(\beta, \bar{\eta}, \dot{\bar{\eta}})+F_{\text {stif }}(\bar{\eta})=F_{\text {ext }},
$$


where $M_{\eta}$ is the discrete mass matrix and $F_{g y r}, F_{\text {stif }}$ and $F_{\text {ext }}$ are the discrete gyroscopic, stiffness, and external generalized forces, respectively. The previous equations need to be solved with the propagation equations of the body-attached reference (the $a$ frame). Several alternatives exist ${ }^{47}$, and in this case, a quaternion representation has been chosen, such that

$$
\begin{aligned}
& \dot{\zeta}=\dot{\zeta}\left(\omega_{a}, \zeta\right)=-\frac{1}{2} \Upsilon\left(\omega_{a}\right) \zeta \\
& \dot{p}_{a}=\dot{p}_{a}\left(\zeta, v_{a}\right)=C^{G a}(\zeta) v_{a}
\end{aligned}
$$

where $\zeta$ is a four-element quaternion vector, $C^{G a}(\zeta)$ is the transformation matrix, via quaternions, from the $a$ frame to the inertial one, and $\Upsilon\left(\omega_{a}\right)$ is given by

$$
\Upsilon\left(\omega_{a}\right)=\left[\begin{array}{cccc}
0 & \omega_{a 1} & \omega_{a 2} & \omega_{a 3} \\
-\omega_{a 1} & 0 & -\omega_{a 3} & \omega_{a 2} \\
-\omega_{a 2} & \omega_{a 3} & 0 & -\omega_{a 1} \\
-\omega_{a 3} & -\omega_{a 2} & \omega_{a 1} & 0
\end{array}\right] .
$$

Extending the set of degrees of freedom given in Eq. (4), the following state vectors are obtained

$$
\begin{gathered}
x_{R}=\left[\begin{array}{llll}
p_{a} & \zeta & v_{a} & \omega_{a}
\end{array}\right]^{T} \\
x_{S}=\left[\begin{array}{ll}
\bar{\eta} & \dot{\bar{\eta}}
\end{array}\right]^{T}
\end{gathered}
$$

Finally, defining a vector that gathers both rigid-body and structural states, the flexible-body equations of motion, Eqs. (5)-(6), can be cast together and solved using a variety of schemes ${ }^{48}$. The resulting equations can also be written as a $1^{\text {st }}$ order discrete state-space equivalent.

\section{Aerodynamic modeling: unsteady vortex lattice method}

The Unsteady Vortex Lattice Method (UVLM) is an effective computational technique to solve potential flow problems about lifting surfaces. The basics of the UVLM algorithm are described in Ref. 42 using an explicit timestepping technique. As in other panel methods, such as the Doublet-Lattice ${ }^{33,34}$, elementary (singularity) solutions are distributed over a surface and the non-penetration boundary condition is imposed at a number of control (collocation) points, leading to a system of algebraic equations. The UVLM is based on thin-airfoil approximation, so both the elementary solutions and the collocation points are placed over the mean surface in lieu of the actual surface, thus effectively ignoring thickness effects.

Lifting surfaces and wakes (the latter obtained as part of the solution procedure) are discretized using vortex rings, i.e., quadrilateral elements composed by discrete vortex segments in a closed loop, along which the circulation strength, $\Gamma_{k}$, is constant (see Figure 2). At the beginning of the motion, only the vortex rings covering the lifting surface exist (bound elements), and collocation points at which flow-tangency will be imposed are associated to them. As the surface moves along its flight path, a force-free wake is formed, shed and convected. In order to accomplish this, each trailing edge bound vortex ring sheds a wake panel, with circulation equal to the trailing edge ring strength at the previous time step. The wake elements are then freely moved according to the local flow velocity, and thus generate a vortex ring lattice representing the shed wake. The circulation strength of the wake rings remains constant as they move away from the lifting surface, but they are allowed to roll up and stretch. Albeit dissipation could also be accounted for by implementing any of various models for wake decay ${ }^{4,49,50}$, this was not regarded necessary for the applications under study. 


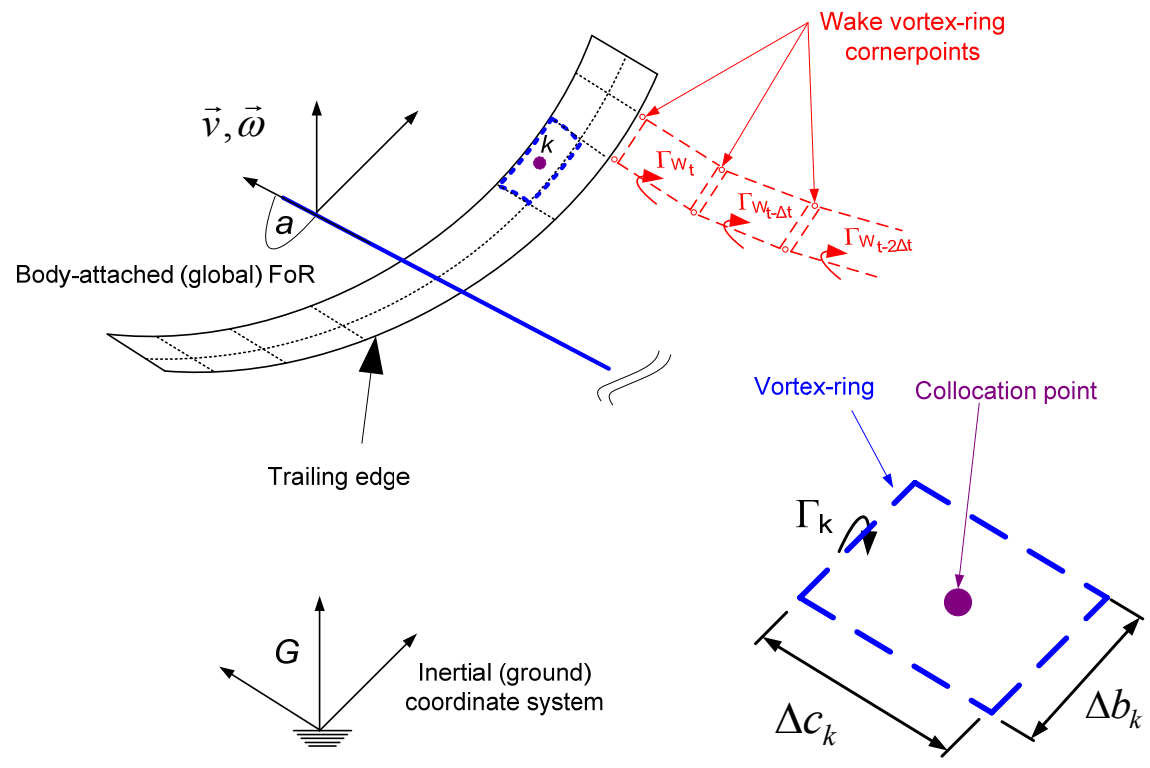

Figure 2. Aerodynamic representation of flexible aircraft: lifting surface and wake discretization using vortex ring elements.

The vorticity distribution of the bound vortex elements is determined by applying the non-penetration boundary condition, requiring a zero velocity component normal to the solid surface at every collocation point $k$

$$
\sum_{l=1}^{K_{b}} a_{k l}\left(\Gamma_{l}\right)_{b}+\sum_{l=1}^{K_{w}} a_{k l}^{*}\left(\Gamma_{l}\right)_{w}+w_{k}=0,
$$

for $l=1,2, \ldots, K_{b}$, where $K_{b}$ is the number of bound vortex rings of the lattice (over the lifting surface); $K_{w}$ is the total number of wake panels; $w_{k}$ stands for the non-vortical contributions to the velocity at collocation point $k$, normal to the surface, encompassing kinematics of the wing (rigid-body motions plus deformations) and free stream; $a_{k l}$ and $a_{k l}^{*}$ are the so-called aerodynamic influence coefficients, corresponding to the bound or wake panels respectively, and they stand for the normal velocity induced by vortex ring $l$ over collocation point $k$, computed by the Biot-Savart law (for unit circulation strength),

$$
\vec{q}=\frac{\Gamma}{4 \pi} \int \frac{d \vec{l} \times \vec{r}}{|\vec{r}|^{3}} .
$$

Here, $\vec{q}$ is the velocity vector induced by a vortex segment of strength $\Gamma ; d \vec{l}$ represents an infinitesimal length along the segment and $\vec{r}$ is the distance between the segment and the collocation point. Note that a vortex ring is composed by four vortex segments.

The numerical evaluation of the Biot-Savart law, Eq. (10), is critical for the accuracy, stability and computational efficiency of the UVLM. There are three major issues that require consideration. First, it is singular at the origin, thereby requiring some kind of treatment to regularize the solution; here, a simple vortex-core model has been implemented. Second, it is the main contributor to the total computational cost, but its influence decays with the square of the distance. Therefore, the burden can be alleviated by neglecting the far-field panels (either on wing or wake) and thus reducing the number of times it is evaluated. Finally, the wake can be truncated as the panels are convected far downstream, ignoring the ones beyond a certain distance from the trailing edge. If all three parameters are chosen correctly, they offer great computational savings with no detrimental effects on accuracy.

Application of the non-penetration boundary condition, Eq. (9), at the $K_{b}$ collocation points leads to a system of algebraic equations, whereby the circulation strength of the bound vortex rings is determined at each time step. 
The aerodynamic loads can be obtained by computing the pressure across each panel, $k$, given by a numerical approximation to the unsteady Bernoulli equation ${ }^{42}$

$$
\Delta p_{k}=\rho\left\{\vec{v}_{k} \cdot\left(\vec{\tau}_{i}\right)_{k} \frac{\left(\Gamma_{i, j}\right)_{b}-\left(\Gamma_{i-1, j}\right)_{b}}{\Delta c_{k}}+\vec{v}_{k} \cdot\left(\vec{\tau}_{j}\right)_{k} \frac{\left(\Gamma_{i, j}\right)_{b}-\left(\Gamma_{i, j-1}\right)_{b}}{\Delta b_{k}}+\frac{\partial \Gamma_{k}}{\partial t}\right\}
$$

where $\vec{v}_{k}$ represents the velocity induced at collocation point $k$ by the wake and the wing motion (downwash); $\left(\vec{\tau}_{i}\right)_{k}$ and $\left(\vec{\tau}_{j}\right)_{k}$ are the panel chordwise and spanwise tangential vectors, respectively; $(\Delta c)_{k}$ and $(\Delta b)_{k}$ are the panel chord and span, and subscripts $(i, j)$ refer to the chordwise and spanwise location of the corresponding bound vortex ring.

The equations governing the unsteady vortex lattice method can be transformed into a discrete state-space equivalent representation. First of all, the non-penetration boundary condition, Eq. (9), can be expressed as

$$
A \boldsymbol{\Gamma}_{b}^{n+1}+A^{*} \boldsymbol{\Gamma}_{w}^{n+1}+\mathbf{w}^{n+1}=0
$$

where $n$ and $n+1$ represent the old and new time-steps; $A=A\left(\boldsymbol{\Gamma}_{b}, \mathbf{X}_{b}\right)$ and $A^{*}=A^{*}\left(\boldsymbol{\Gamma}_{b}, \boldsymbol{\Gamma}_{w}, \mathbf{X}_{b}, \mathbf{X}_{w}\right)$ are the wing-wing and wake-wing aerodynamic influence matrices, respectively; $\boldsymbol{\Gamma}_{b}$ and $\boldsymbol{\Gamma}_{w}$ correspond to the bound and wake vorticity vectors storing the circulation strengths of the $K_{b}$ and $K_{b}$ vortex rings, respectively, and $\mathbf{X}_{b}$ and $\mathbf{X}_{w}$ stand for the coordinates of the grid points on the aerodynamic lattice; finally, $\mathbf{w}=\mathbf{w}\left(\mathbf{X}_{b}, \dot{\mathbf{X}}_{b}\right)$ is the vector with the normal components of the non-vortical induced velocities.

As the vehicle moves, the wake is shed along the trailing edge of the wings. The closing segment of the new wake panel is located along the position of the trailing edge at the current time step, but it inherits the vorticity of the (bound) trailing edge panel at the previous time step. The wake panels that were shed previously are (optionally) rolled up according to the local flow velocity, and they retain their circulation strength as they are moved. These wake shedding and roll-up operations can be written in discrete state-space fashion as

$$
\begin{aligned}
& \boldsymbol{\Gamma}_{w}^{n+1}=B \boldsymbol{\Gamma}_{b}^{n}+B^{*} \boldsymbol{\Gamma}_{w}^{n} \\
& \mathbf{X}_{w}^{n+1}=C \mathbf{X}_{b}^{n+1}+C^{*} \mathbf{X}_{w}^{n}+D \boldsymbol{\Gamma}_{b}^{n+1}+D^{*} \boldsymbol{\Gamma}_{w}^{n+1},
\end{aligned}
$$

where $B, B^{*}, C$ and $C^{*}$ are very sparse matrices - note that if any dissipation model was to be assumed for the wake, this should be included in $B^{*}$. In the absence of wake roll-up (or prescribed wake) $D=D^{*}=0$, but if it is implemented, $D=D\left(\mathbf{X}_{b}, \mathbf{X}_{w}\right)$ and $D^{*}=D^{*}\left(\mathbf{X}_{w}\right)$, and their sparsity will be dependent upon the definition of the cut-off distance for the far-field approximation. The time step at which $\boldsymbol{\Gamma}_{b}, \boldsymbol{\Gamma}_{w}, \mathbf{X}_{b}, \dot{\mathbf{X}}_{b}$ and $\mathbf{X}_{w}$ are evaluated for the computation of the matrices $A^{(*)}-D^{(*)}$ in Eqs. (12) and (13) will depend on the selected integration scheme.

Hence, for the case of prescribed kinematics and wing deformations (given $\mathbf{X}_{b}$ and $\dot{\mathbf{X}}_{b}$ ), Eqs. (12) and (13) define a discrete state-space problem with state vector given as

$$
x_{A}=\left\{\begin{array}{l}
\boldsymbol{\Gamma}_{b} \\
\boldsymbol{\Gamma}_{w} \\
\mathbf{X}_{w}
\end{array}\right\}
$$

leading to

$$
\left\{\begin{array}{l}
\boldsymbol{\Gamma}_{b} \\
\boldsymbol{\Gamma}_{w} \\
\mathbf{X}_{w}
\end{array}\right\}^{n+1}=\left[\begin{array}{ccc}
-A^{-1} A^{*} B & -A^{-1} A^{*} B^{*} & 0 \\
B & B^{*} & 0 \\
D & D^{*} & C^{*}
\end{array}\right]\left\{\begin{array}{l}
\boldsymbol{\Gamma}_{b} \\
\boldsymbol{\Gamma}_{w} \\
\mathbf{X}_{w}
\end{array}\right\}^{n}+\left\{\begin{array}{c}
-A^{-1} \mathbf{w}^{n+1} \\
0 \\
0
\end{array}\right\} .
$$


If the wake roll-up is not taken into account, the simplified state variable would not include $\mathbf{X}_{w}$, i.e., $x_{A}=\left[\begin{array}{ll}\boldsymbol{\Gamma}_{b} & \boldsymbol{\Gamma}_{w}\end{array}\right]^{T}$, and the formulation presented by Hall ${ }^{51}$ would be recovered for the state-space equivalent of the UVLM. Finally, Eq. (11) provides the aerodynamic loads as an output of the system, which can be also expressed in discrete time as

$$
\mathbf{F}_{\text {aero }}^{n+1}=F_{a 1} \boldsymbol{\Gamma}_{b}^{n+1}+F_{a 2} \boldsymbol{\Gamma}_{b}^{n},
$$

where $F_{a 1}=F_{a 1}\left(\mathbf{X}_{b}, \dot{\mathbf{X}}_{b}\right)$ and $F_{a 2}=F_{a 2}\left(\mathbf{X}_{b}\right)$ transform the pressure drop across the panels into forces and moments. They are very sparse matrices, since only the effect of a vortex ring on itself contributes to the aerodynamic loads.

\section{Full aeroelastic and flight dynamics model: tightly-coupled state-space representation}

The previous flexible-body and aerodynamic models will be used to represent the complete dynamics of a flexible HALE vehicle. As the structural model is based on beams (curves in space) and the aerodynamic lattice is distributed over a lifting surface (see Figure 3), a mapping procedure is required between both meshes.

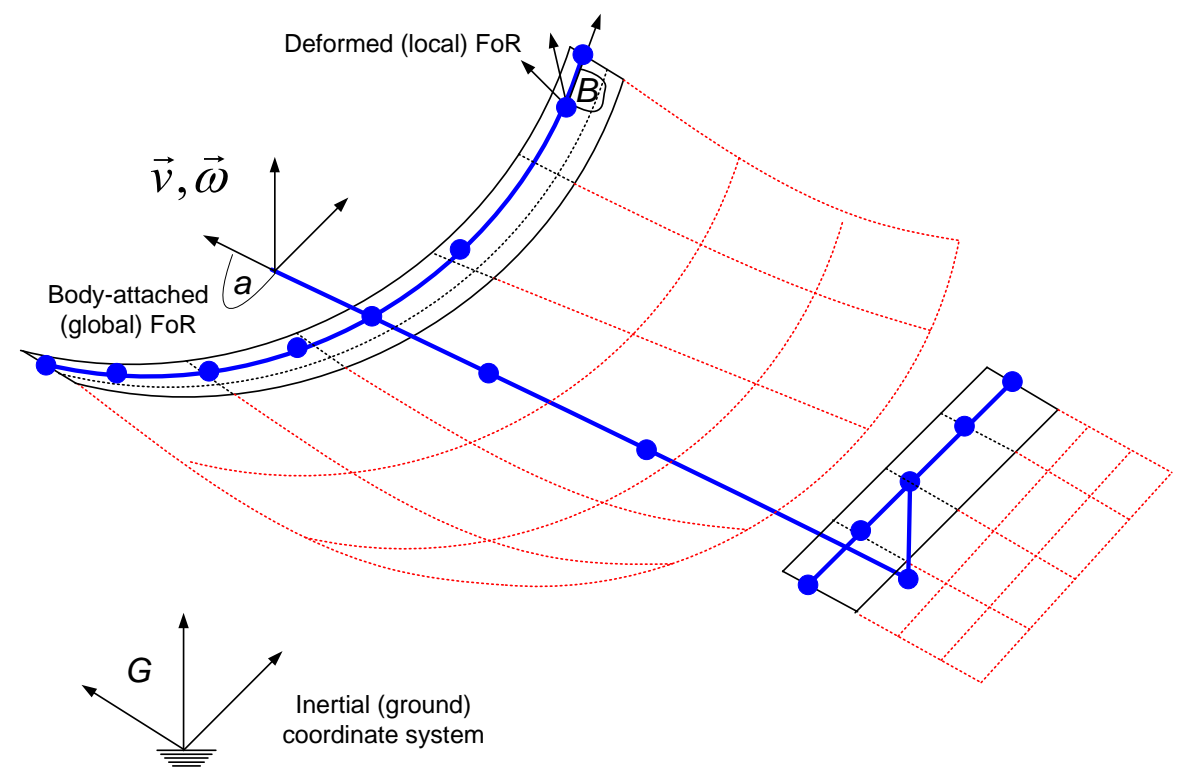

Figure 3. Full representation of flexible aircraft: beam-like structure, vortex-ring lattice and rigid-body motions.

Firstly, displacements and rotations of the beam nodes have to be transformed to deformations of the grid points of the aerodynamic lattice. This is done with the assumption that airfoils remain rigid under wing deformations. Vortex-ring cornerpoints or collocation points are expressed in the aerodynamic coordinate system, defined independently, but can be mapped to and from the other reference frames straightforwardly.

Secondly, it is necessary to transform the pressure difference computed in Eq. (11) to forces and moments acting upon the beam nodes. For that purpose, it is assumed that they can be approximated by isolated aerodynamic loads applied in the center of the leading segment of each vortex-ring. The application points of the forces and moments that arise can be mapped and transformed to the deformed frame, $B$. The loads expressed in their components in the deformed reference frame are finally lumped into the nodes of the deformed beam.

Through the above mapping procedures, the coupling of aerodynamic and structural models for a full aeroelastic and flight dynamics characterization of the vehicle can be tackled. This can be done in different fashions, and a state-space tightly-coupled formulation is presented here. For this purpose, the state variables of the flexible-body model, Eq. (8), are augmented with the aerodynamic model, Eq. (14), leading to 


$$
x=\left[\begin{array}{lll}
x_{R} & x_{S} & x_{A}
\end{array}\right]^{T}=\left[\begin{array}{lllllllllll}
p_{a} & \zeta & v_{a} & \omega_{a} & \mathbf{R}_{a} & \dot{\mathbf{R}}_{a} & \boldsymbol{\Psi} & \dot{\Psi} & \boldsymbol{\Gamma}_{b} & \boldsymbol{\Gamma}_{w} & \mathbf{X}_{w}
\end{array}\right]^{T} .
$$

The resulting discrete state-space equations on motion can be cast into

$$
\left\{\begin{array}{l}
x_{R} \\
x_{S} \\
x_{A}
\end{array}\right\}^{n+1}=\left[\begin{array}{ccc}
\Lambda_{R} & \Lambda_{R S} & \Lambda_{R A} \\
\Lambda_{S R} & \Lambda_{S} & \Lambda_{S A} \\
\Lambda_{A R} & \Lambda_{A S} & \Lambda_{A}
\end{array}\right]\left\{\begin{array}{l}
x_{R} \\
x_{S} \\
x_{A}
\end{array}\right\}+\left\{\begin{array}{l}
\lambda_{R} \\
\lambda_{S} \\
\lambda_{A}
\end{array}\right\} u^{n+1}
$$

where, $\Lambda_{R}, \Lambda_{R S}, \Lambda_{S R}$ and $\Lambda_{S}$ can be obtained from the state-space equivalent of the flexible-body dynamics equations, Eqs. (5)-(6), and are, in general, function of the state vector; the influence of the aerodynamics over the flight dynamics and elastic beam is described, respectively, in the matrix functionals $\Lambda_{R A}$ and $\Lambda_{S A}$, which transform the aerodynamic loads from the aerodynamic grid to forces and moments acting upon the flexible aircraft, Eqs. (5) and (16); any other applied load is transferred to the forcing vector, $u ; \Lambda_{A}$ is obtained from the state-space equivalent of the aerodynamic UVLM, Eq. (15); $\Lambda_{A R}$ represents the influence of the rigid-body motions on the aerodynamic model, mapping both positions and velocities from the beam nodes to the aerodynamic lattice; analogously, $\Lambda_{A S}$ accounts for the influence of the deformation of the beam (wing); both $\Lambda_{A R}$ and $\Lambda_{A S}$ contribute to $\mathbf{w}$ in Eq. (15).

In Eq. (18), $\lambda_{R}, \lambda_{S}$ and $\lambda_{A}$ correspond to the input or control matrices, and $u$ represents the forcing functions, which will encompass inputs such as thrust provided by the engines, weight of the various elements of the vehicle, viscous drag, control surfaces, cockpit controls, etc. Again, the time step at which the matrix functionals $\Lambda_{i}$ and $\lambda_{i}$ are evaluated in Eq. (18) will be determined by the chosen integration scheme. Also note that the formulation presented so far corresponds to the open-loop characterization of the flexible aircraft.

\section{Aeroelastic model with prescribed aircraft dynamics}

For the numerical studies in this work (Section VI), the rigid-body motions of the aircraft are prescribed. As a result, the equations presented in previous sections can be simplified. When the motion of the reference frame, $a$, is predefined, the vector comprising the rigid-body motions, $x_{R}$, can then be removed from the state of the system and Eq. (5) is written as ${ }^{18}$

$$
M_{t}(\bar{\eta}) \ddot{\bar{\eta}}+m_{a}(\bar{\eta})\left\{\begin{array}{c}
\dot{v}_{a} \\
\dot{\omega}_{a}
\end{array}\right\}+F_{g y r}^{s t}\left(\bar{\eta}, \dot{\bar{\eta}}, v_{a}, \omega_{a}\right)+F_{s t i f f}^{s t}(\bar{\eta})=F_{e x t}^{s t},
$$

where, in this case, $M_{t}$ and $m_{a}$ are submatrices of the tangent mass matrix in Eq. (5). The aerodynamic model does not change and the aeroelastic state vector will then be given by

$$
x=\left[\begin{array}{ll}
x_{S} & x_{A}
\end{array}\right]^{T}=\left[\begin{array}{lllllll}
\mathbf{R}_{a} & \dot{\mathbf{R}}_{a} & \boldsymbol{\Psi} & \dot{\boldsymbol{\Psi}} & \boldsymbol{\Gamma}_{b} & \boldsymbol{\Gamma}_{w} & \mathbf{X}_{w}
\end{array}\right]^{T},
$$

leading to the discrete state-space equations

$$
\left\{\begin{array}{l}
x_{S} \\
x_{A}
\end{array}\right\}^{n+1}=\left[\begin{array}{cc}
\Lambda_{S} & \Lambda_{S A} \\
\Lambda_{A S} & \Lambda_{A}
\end{array}\right]\left\{\begin{array}{l}
x_{S} \\
x_{A}
\end{array}\right\}^{n}+\left\{\begin{array}{l}
\lambda_{S} \\
\lambda_{A}
\end{array}\right\} u^{n+1} .
$$

The different matrix functionals in Eq. (21) will account for the prescribed rigid-body motions, which affect both the structure and the aerodynamics.

Finally, even though a coupled state-space formulation has been presented, which is particularly useful for stability analyses, it is also possible to retain the second order version of the flexible-body equations of motion, Eq. (5), and thus use specific algorithms tailored for time integration of structural dynamics problem ${ }^{48}$. This is the approach that has been followed for the numerical studies of this work: the aerodynamics are marched in time using 
a first-order forward Euler scheme, whereas the structural model follows an implicit Newmark method including a Newton-Raphson iterative scheme. Both models are coupled by the mapping procedure described in Section V.

The aerodynamic UVLM has been implemented in MATLAB, whereas the structural model was written in FORTRAN. For the interface between the two codes, MATLAB allows to create dynamically linked subroutines produced from $\mathrm{C}, \mathrm{C}++$ or FORTRAN source code that, when compiled, can be read and run from within MATLAB in the same way as MATLAB M-files or built-in functions ${ }^{52}$. Hence, MEX-files have been created for the relevant FORTRAN routines, and the coupled model is run in MATLAB.

\section{Numerical studies}

This section presents several numerical studies using the present coupled model for flexible aircraft dynamics. The structural and aerodynamic models had been previously validated independently, and various studies have already been reported in Ref. 53. Hence, only the full coupled model will be considered here. First of all, the flutter speed of the Goland wing is computed for code verification purposes using different procedures. Next, a representative HALE wing model is considered, and results are obtained and compared against other models. Finally, the influence of wake interference and rollup is investigated for a stiffened version of the above HALE aircraft.

\section{A. Goland wing}

The Goland wing is a relatively stiff and small aspect-ratio wing that has long been used as a benchmark in aeroelastic calculations, since Goland ${ }^{54}$ first analyzed it. Its flutter onset point has been computed by many authors $^{54,55}$ and this will be used for the verification of the present model. Table 1 describes the relevant properties of the Goland wing, the discretization parameters and the flight conditions of this analysis.

Table 1. Goland wing simulation parameters

\begin{tabular}{lrlr}
\hline \multicolumn{1}{c}{ Goland wing properties } & & \multicolumn{1}{c}{ Flight conditions } \\
Chord & $1.8288 \mathrm{~m}$ & Air density & $1.020 \mathrm{~kg} / \mathrm{m}^{3}$ \\
Semi-span & $6.096 \mathrm{~m}$ & Angle of attack & $0.05 \mathrm{deg}$ \\
Elastic axis (from leading edge) & $33 \%$ chord & & Discretization \\
Center of gravity (from leading edge) & $43 \% \mathrm{chord}$ & & 10 \\
Mass per unit length & $35.71 \mathrm{~kg} / \mathrm{m}$ & & 4 \\
$\begin{array}{l}\text { Moment of inertia (around elastic } \\
\text { axis) }\end{array}$ & $8.64 \mathrm{~kg} \cdot \mathrm{m}$ & Number of beam elements \\
Torsional rigidity & $0.99 \times 10^{6} \mathrm{~N} \cdot \mathrm{m}^{2}$ & Number of chordwise panels & 10 \\
Bending rigidity & $9.77 \times 10^{6} \mathrm{~N} \cdot \mathrm{m}^{2}$ & Number of spanwise panels & 0.3 \\
Chordwise (bending) rigidity & $9.77 \times 10^{8} \mathrm{~N} \cdot \mathrm{m}^{2}$ & Time-step $\left(\Delta t^{*}=\Delta t \times V_{\infty} / c_{\text {wing }}\right)$ & \\
\hline \hline
\end{tabular}

The onset of instability can be computed in several ways. In this case, two different approaches have been followed. First of all, the flutter speed can be obtained by pre-computing the frequency-domain aerodynamics for the relevant vibration modes and then investigate the stability of the resulting system. In this case, the aerodynamic UVLM and the structural beam model are effectively decoupled, and the procedure consists of the following steps:

1) The first few linear vibration modes of the structure are computed.

2) These modes are prescribed in purely aerodynamic time-domain simulations, in order to obtain the corresponding harmonic forces at given frequencies.

3) Time-domain solutions are converted into frequency-domain by computing the transfer function of the relevant aerodynamic magnitude.

4) By scanning different frequencies in the range of interest, a set of frequency-domain solutions is obtained, which can be cast into the so-called matrix of Generalized Aerodynamic Forces (GAF). 
5) This GAF matrix can be approximated by a Rational Function Approximation (RFA) ${ }^{56}$ with a number of lag parameters (poles), and appended to the structural dynamic equations of motion in state-space fashion - the reader is referred to Ref. 57 for a more detailed description.

6) Once the state-space equivalent of the reduced aeroelastic model is obtained, the stability analysis is straightforward, and the neutral stability boundary will be determined when the real part any of the eigenvalues of the system changes sign from negative to positive.

Using this procedure it has been found that the first torsion mode becomes unstable at a velocity of $V_{f}=177 \mathrm{~m} / \mathrm{s}$, which corresponds to a frequency of $\omega_{f}=68 \mathrm{rad} / \mathrm{s}$.

Note that following this procedure time-domain simulations are performed to generate GAFs in the frequencydomain, and these are then converted back to time-domain using the RFA. The intermediate step could be omitted by using the Eigensystem Realization Algorithm (ERA) ${ }^{58}$, which transforms an impulse response into state-space form directly. Nevertheless, the use of neither ERA nor similar direct approaches was found necessary for this work.

The flutter speed can also be estimated from time-domain aeroelastic simulations. For that purpose, the Goland wing is started from rest at an undeformed position and the coupled aeroelastic model is marched in time in a linear dynamic simulation. Different free-stream velocities $\left(V_{\infty}\right)$ can be prescribed until the flutter onset is found. Figure 4 depicts the time-histories of the wing-tip deflection at different air-speeds. In order to illustrate the instability, examples of decaying $\left(V_{\infty}=160 \mathrm{~m} / \mathrm{s}\right)$ and growing $\left(V_{\infty}=170 \mathrm{~m} / \mathrm{s}\right)$ periodic responses are presented together with the neutral speed $\left(V_{f}=165 \mathrm{~m} / \mathrm{s}\right)$. At this point, the corresponding flutter frequency is $\omega_{f}=69 \mathrm{rad} / \mathrm{s}$.

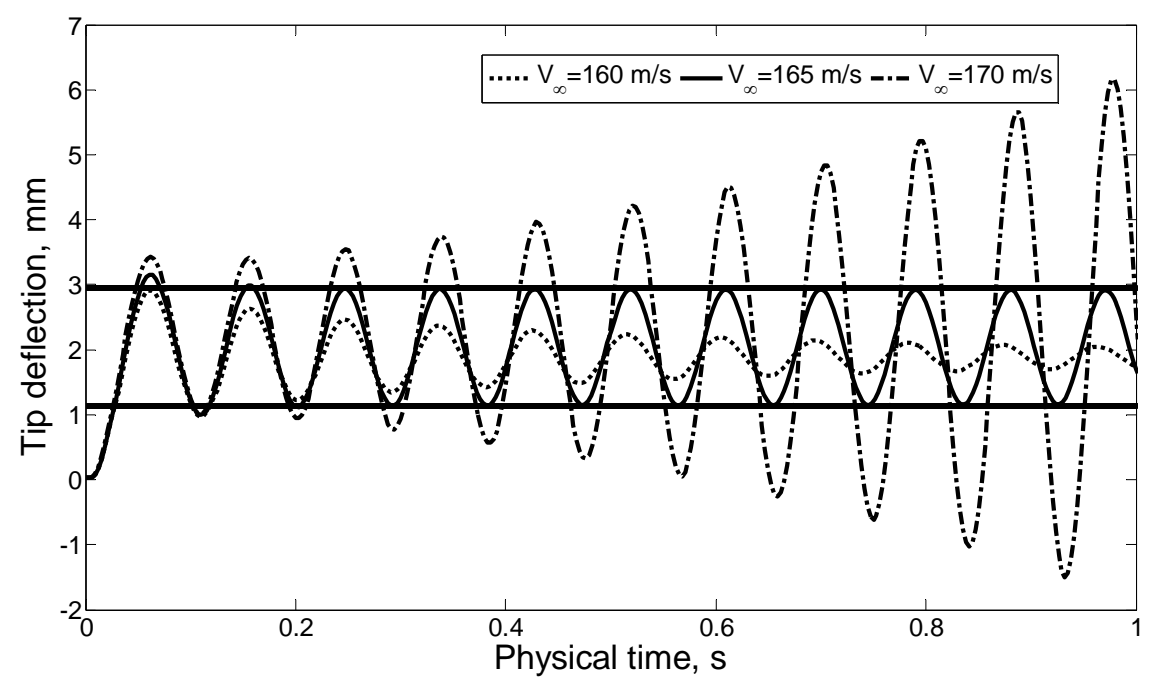

Figure 4. Goland wing-tip time-history at various free-stream velocities for $\alpha=0.05$ deg. Damped, undamped, and neutral solutions.

By using the methodologies described above, two different estimates of the flutter speed and frequency have been obtained, and are shown in Table 2. They are compared to results published in the literature and, as can be observed, the agreement is remarkable. Hence, this quantitative exercise with the Goland wing verifies the present implementation of the coupled aeroelastic model described in Section V.

Table 2. Flutter speed for the Goland wing

\begin{tabular}{llc}
\hline \hline Author & Model & Flutter speed [m/s] \\
\hline Goland $^{54}$ & --- & 175.6 \\
Wang et al $^{55}$ & ZAERO $^{\circledR}$ (Doublet-Lattice, frequency domain) & 174.3 \\
Wang et al $^{55}$ & Intrinsic beam + UVLM & 163.8 \\
Present time-domain & Displacement beam + UVLM & 165 \\
Present RFA & Displacement beam + UVLM + RFA & 177 \\
\hline \hline
\end{tabular}

12

American Institute of Aeronautics and Astronautics 


\section{B. Static aeroelastic analysis of flexible HALE model aircraft}

Next, a representative HALE-like model aircraft has been considered. The vehicle consists of a very high aspectratio main wing and a horizontal tail-plane. This numerical test-case was first introduced by Patil et al ${ }^{3}$, using intrinsic beam equations and a 2-D finite-state air-loads model. The geometry of the aircraft is shown in Figure 5 and the relevant properties are listed in Table 3.

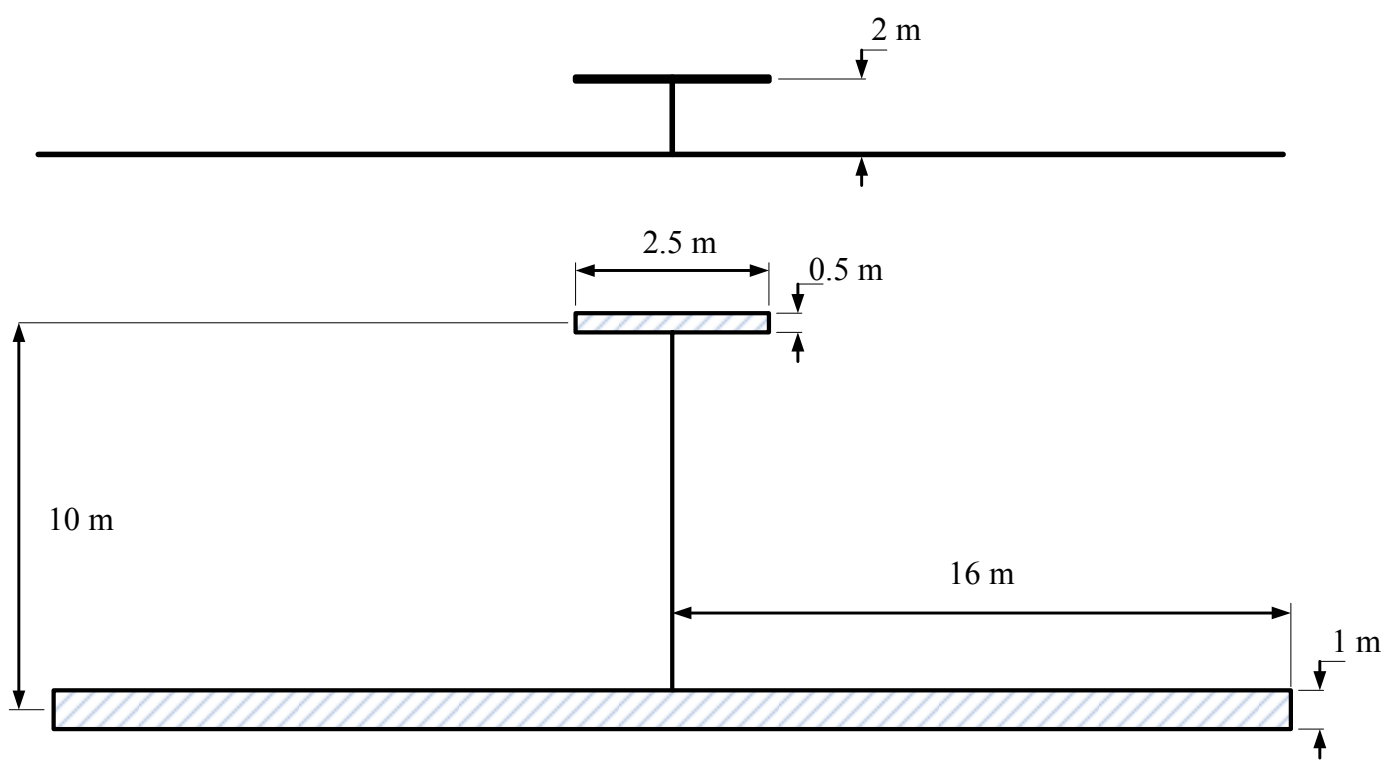

Figure 5. HALE model aircraft geometry. Large aspect-ratio main wing and rigid tail-plane. Front and top views.

Table 3. HALE model aircraft properties

\begin{tabular}{lcc}
\hline \hline & Main wing & Tail \\
\hline Chord & $1 \mathrm{~m}$ & $0.5 \mathrm{~m}$ \\
Semi-span & $16 \mathrm{~m}$ & $2.5 \mathrm{~m}$ \\
Elastic axis (from leading edge) & $50 \%$ chord & N/A \\
Center of gravity (from leading edge) & $50 \%$ chord & $50 \%$ chord \\
Mass per unit length & $0.75 \mathrm{~kg} / \mathrm{m}$ & $0.08 \mathrm{~kg} / \mathrm{m}$ \\
Moment of inertia (around elastic axis) & $0.1 \mathrm{~kg} \cdot \mathrm{m}$ & $0.01 \mathrm{~kg} \cdot \mathrm{m}$ \\
Torsional rigidity & $2 \times 10^{4} \mathrm{~N} \cdot \mathrm{m}^{2}$ & $\infty$ \\
Bending rigidity & $1 \times 10^{4} \mathrm{~N} \cdot \mathrm{m}^{2}$ & $\infty$ \\
Chordwise (bending) rigidity & $4 \times 10^{6} \mathrm{~N} \cdot \mathrm{m}^{2}$ & $\infty$ \\
\hline Mass of payload & $0.0899 \mathrm{~kg} / \mathrm{m}^{3}(20 \mathrm{~km}$ altitude $)$ \\
Air-density & $50 \mathrm{~kg}$ & \\
\hline \hline
\end{tabular}

Published figures are compared to results obtained using the present coupled aeroelastic model. First, Figure 6 shows the static trim angle of attack at various flight speeds, for rigid and flexible versions of the HALE vehicle under study. Even though the differences are not dramatic, the present model yields higher trim angles for both cases. This is due to the fact that the three-dimensional aerodynamic effects are being fully accounted for. Figure 7 depicts the total lift ratio between a flexible and a rigid aircraft at various free-stream velocities. In this case, a constant angle of attack has been prescribed and linear and nonlinear computations have been carried out. As 
already observed in Ref. 3, the linear model substantially overestimates the lift, which would lead to an incorrect prediction of the flight envelope. The discrepancy between the results provided by the different models increases with velocity, but it falls within reasonable margins, showing that the 2-D aerodynamics give a good approximation to the aircraft response. More details in a similar comparison between 2-D and 3-D aerodynamic models for very flexible wings were presented in Ref. 53. Finally, following the same time-domain solution process described in Section A for the Goland wing, the flutter speed and frequency have been found to be $V_{f}=33.5 \mathrm{~m} / \mathrm{s}$ and $\omega_{f}=22.4$ $\mathrm{rad} / \mathrm{s}$, whereas in Ref. 3 it is $V_{f}=32.21 \mathrm{~m} / \mathrm{s}$ and $\omega_{f}=22.61 \mathrm{rad} / \mathrm{s}$. Good agreement has therefore been found between the current model and Ref. 3.

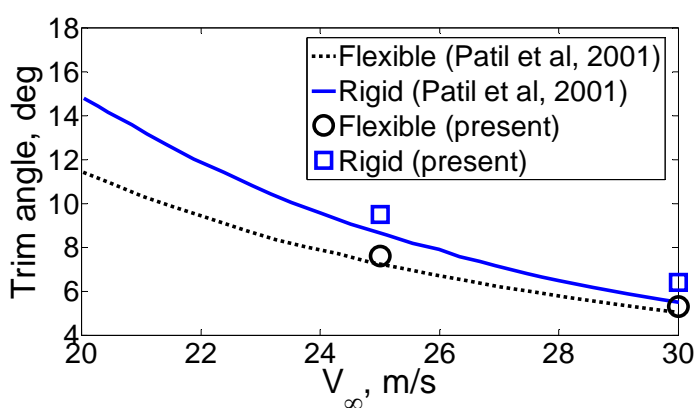

Figure 6. Variation of trim angle with flight speed.

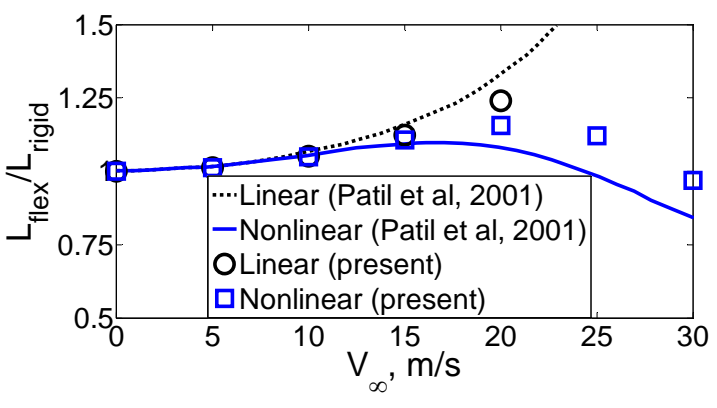

Figure 7. Flexible-to-rigid aircraft at $\alpha=5 \mathrm{deg}$.

\section{Wake interference on stiffened HALE model aircraft}

In order to explore the influence of wake interference on the dynamics of a HALE aircraft, a stiffened version of the vehicle described in Table 3 has been considered. The stiffness matrix has been multiplied by 5 so that the static deformation of the wing-tip of the trimmed aircraft is of the order of $10 \%$ its half-span. The rest of the parameters are retained without changes. The characteristics of this stiffened version of the HALE aircraft are listed in Table 4. Figure 8 depicts the shape of the deformed main wing at different trim conditions.

\section{Table 4. Stiffened HALE aircraft}

\begin{tabular}{lc}
\hline \hline Flutter speed & $74 \mathrm{~m} / \mathrm{s}$ \\
$1^{\text {st }}$ bending frequency & $0.81 \mathrm{~Hz}$ \\
$1^{\text {st }}$ torsion frequency & $11.05 \mathrm{~Hz}$ \\
Trim angle of attack at $V_{\infty}=40 \mathrm{~m} / \mathrm{s}$ & $2.5 \mathrm{deg}$ \\
Tip deflection at $V_{\infty}=40 \mathrm{~m} / \mathrm{s}$ & $1.73 \mathrm{~m}$ \\
Trim angle of attack at $V_{\infty}=25 \mathrm{~m} / \mathrm{s}$ & $7.2 \mathrm{deg}$ \\
Tip deflection at $V_{\infty}=25 \mathrm{~m} / \mathrm{s}$ & $1.65 \mathrm{~m}$ \\
\hline \hline
\end{tabular}

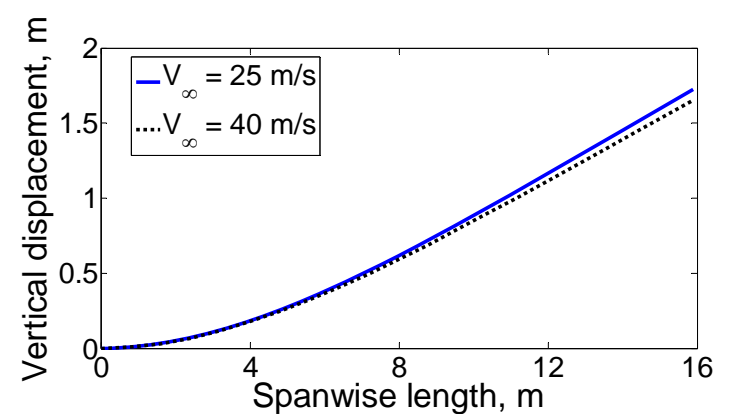

Figure 8. Deformed wing shape at different trim conditions.

Different motions have been prescribed for this aircraft so that the influence of the wake over the trailing tailplane could be investigated, and its impact on the handling capabilities of the vehicle assessed. To that goal, three different types of motion have been prescribed around the nonlinear static trim condition: plunging, pitching, and combined plunging-pitching for a constant incidence angle. They are described in more detail in the following subsections.

In order to evaluate the significance of the wake effects on the tail, the evolution of its lift coefficient has been monitored. The results obtained using the present coupled aeroelastic model are compared to other approximations to the lift coefficient slope $\left(C_{L, \alpha}\right)$. One simplification is to assume that the tail is a wing flying alone with a steady wake behind, represented by horseshow vortices, for instance (it will be referred to as the "flying tail" approximation). A more sophisticated approach would be to consider the whole aircraft flying steadily at the trim condition, accounting for both the wake shed by the deformed main wing and the tail itself ("full aircraft" 
approximation). In both situations, the lift coefficient slope can be obtained by perturbing the original state by an infinitesimal amount, and computing the resultant variation in lift.

In the following subsections, these approximate values will be shown along with the results obtained via the present model, for the three motions mentioned above. Results are presented for two different flight speeds, namely $V_{\infty}=40 \mathrm{~m} / \mathrm{s}$ and $V_{\infty}=25 \mathrm{~m} / \mathrm{s}$, for which the trim angles of attack are, respectively, $\alpha_{0}=2.5 \mathrm{deg}$ and $\alpha_{0}=7.2 \mathrm{deg}$. The approximate lift coefficient slopes are shown in Table 5.

Table 5. Approximate lift coefficient slopes at different trim conditions

\begin{tabular}{ccc}
\hline \hline Free-stream velocity, $V_{\infty}$ & Flying tail approximation, $\left(C_{L, \alpha}\right)_{1}$ & Full aircraft approximation, $\left(C_{L, \alpha}\right)_{2}$ \\
\hline $40 \mathrm{~m} / \mathrm{s}$ & $4.93 \mathrm{rad}^{-1}$ & $4.58 \mathrm{rad}^{-1}$ \\
$25 \mathrm{~m} / \mathrm{s}$ & $4.81 \mathrm{rad}^{-1}$ & $4.43 \mathrm{rad}^{-1}$ \\
\hline \hline
\end{tabular}

For the present aeroelastic model the discretization shown in Table 6 has been used, which is enough to guarantee that the dynamics of the problem and the variations of the tail-lift are well captured.

Table 6. Discretization for the HALE model aircraft in the wake-interference analysis

\begin{tabular}{lcc}
\hline \hline & Main wing & Tail \\
\hline Number of beam elements & 20 & 8 \\
Number of chordwise panels & 6 & 4 \\
Number of spanwise panels & 20 & 8 \\
\hline Non-dimensional time-step $\left(\Delta t^{*}=\Delta t \times V_{\infty} / c_{\text {wing }}\right)$ & 0.5 \\
\hline \hline
\end{tabular}

\section{Pure plunging}

First of all, a pure harmonic plunging motion of the HALE model has been analyzed. In this case, the angle of attack of the aircraft remains constant at the trim angle, but the whole aircraft experiences a change in altitude given by

$$
h=A_{h} \sin \left(\omega_{h} t\right) .
$$

As a result, the induced angle of attack due to this plunging motion changes the effective incidence angle of the vehicle, which will in turn modify the aerodynamic loads over the main wing and they will deflect correspondingly around the static deformed position. The induced angle of attack due to this pitching motion is $\alpha_{\text {ind }}=-\tan ^{-1}\left(A_{h} \omega_{h} / V_{\infty}\right)$. The frequency of the oscillation is selected so that it is close enough to the $1^{\text {st }}$ bending mode of the main wing, and has been set to $\omega_{h}=5 \mathrm{rad} / \mathrm{s}$. The amplitude of the plunging motion has been chosen in order to guarantee center of gravity of the deformed aircraft remains at the same altitude, and hence depends on the flight speed.

Two different trim conditions have been studied. First of all, free-stream velocity of $V_{\infty}=40 \mathrm{~m} / \mathrm{s}$ has been assumed, which corresponds to a trim angle of $\alpha_{0}=2.5 \mathrm{deg}$. The deformed main wing in static equilibrium is illustrated in Figure 8. The plunging amplitude that corresponds to this condition is $A_{h}=0.7894 \mathrm{~m}$. Figure 9 shows the lift coefficient of the tail-plane obtained via the present model, compared to the two different approximations to $C_{L, \alpha}$ described above - note that for this motion, the lift coefficient is given by $C_{L}=C_{L, \alpha} \times\left[\alpha_{0}-\tan ^{-1}\left(\dot{h} / V_{\infty}\right)\right]$, where $C_{L, \alpha}$ will be the relevant value of Table 5 . The left-hand-side plot illustrates the evolution in time for a complete plunging cycle, whereas the one in the right-hand side presents the lift coefficient as a function of the vertical displacement of the whole aircraft.

It can be seen that, for this case, the more elaborate approximation provides a better agreement with respect to the solution of the coupled model. For the flying tail approximation, the presence of the wake of the main wing is not taken into account, and hence it overestimates the maximum value of lift when the aircraft is descending and the wake nears the tail. For the full aircraft approximation, the maximum value is more closely estimated, with a slight mismatch for the minimum value. The approximation seems to yield reasonably satisfactory results in this case. 


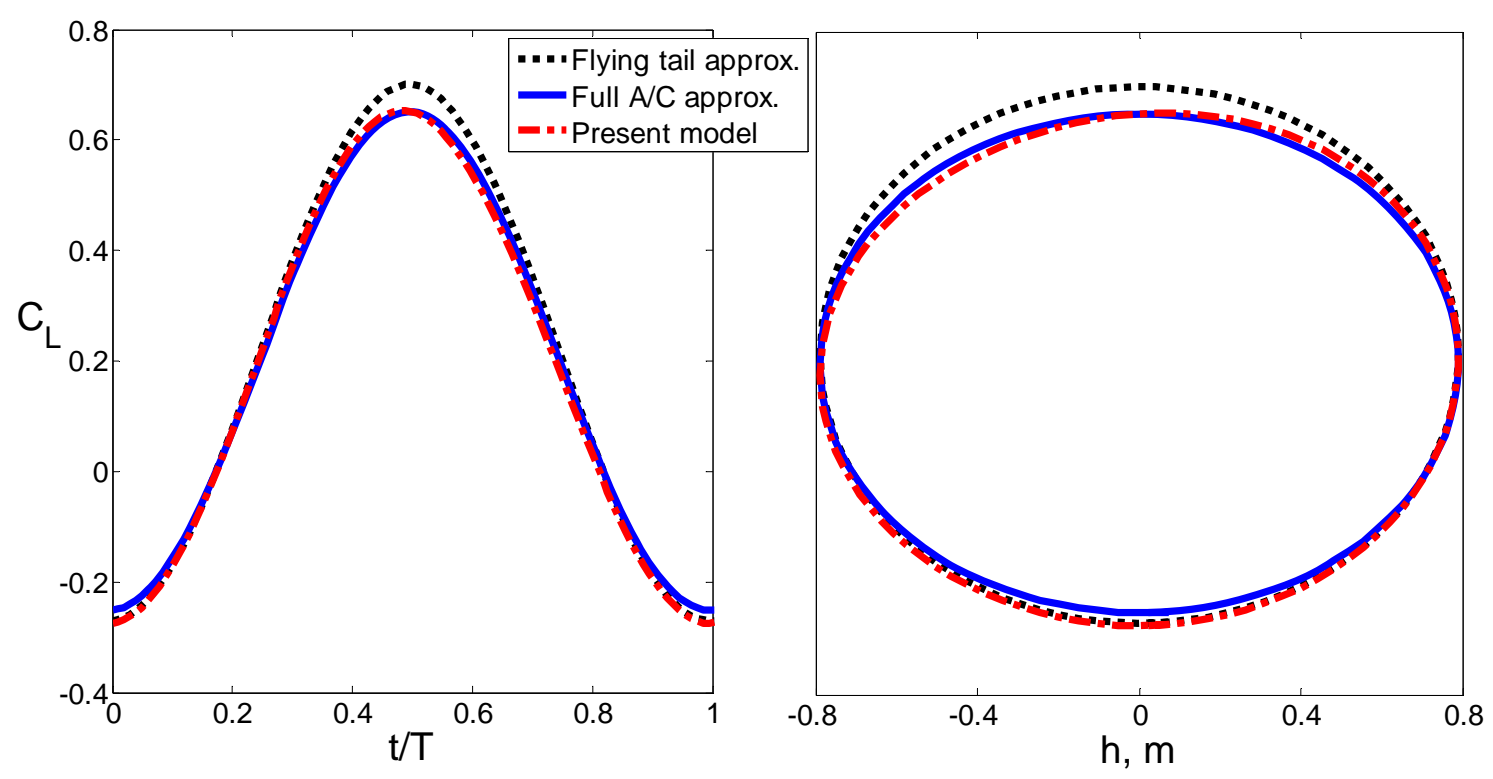

Figure 9. Tail-lift coefficient for an aircraft in plunging motion. $V_{\infty}=40 \mathrm{~m} / \mathrm{s}, \alpha_{0}=2.5 \mathrm{deg}, A_{h}=0.7894 \mathrm{~m}$, and $\omega_{h}=5 \mathrm{rad} / \mathrm{s}$.

Next, a free-stream velocity of $V_{\infty}=25 \mathrm{~m} / \mathrm{s}$ has been considered, which leads to the deformed static shape shown in Figure 8 with a trim angle of of $\alpha_{0}=7.2 \mathrm{deg}$. In this case, the plunging amplitude is of $A_{h}=0.7487 \mathrm{~m}$. Results for the tail-plane lift coefficient are shown in Figure 10, illustrating time-evolution during a period and dependence on the plunging motion. The most notable feature of this case is the presence of discontinuities in the values obtained through the coupled aeroelastic model. The most obvious one occurs near maximum lift, but there is another jump, not so dramatic, at around $t / T=0.75$. These are caused by the tail crossing the wake shed by the main wing, which will be called subsequently "interaction". Note that "interaction" implies a direct wake-tail collision, as opposed to "interference", which refers to the influence of a wake in the proximity of a lifting-surface.

The first interaction occurs as the aircraft descends and passes through nearly the original position and the taillift experiences a sudden increase. The second interaction, in turn, takes place as the aircraft has already started climbing, and the resulting effect on the lift is an almost instantaneous drop. While the alternative methods proposed for the lift computation are unable to predict these jumps, a refined enough temporal and spatial discretization of the UVLM allows to capture them.

Another noteworthy consideration is that the simplest approximation (labeled as "flying tail") provides results that are closer to the 3-D aerodynamic model, even though the mismatch is still considerable. Hence, none of the alternatives is successful in fully capturing the behavior. The discrepancies with respect to the UVLM are significant and the wake-surface encounters are entirely missed.

The sudden changes experienced by the tail-lift are representative examples of wake-airfoil collisions, similar to Blade-Vortex Interactions (BVI) in helicopter rotors ${ }^{35-39}$. Even though it is beyond the scope of this paper to study the specific features of the vortex-body impingement in detail, the UVLM provides a first estimate of the effect of this interference ${ }^{43}$. Observing the first and most notorious jump in Figure 10, it can be inferred that there is a gradual decrease in lift as the wake approaches the trailing tail, followed by a rapid rise when the nearest position is reached, which is consistent with the behavior described and illustrated for a BVI in Refs. 36 and 38, for instance. The UVLM should be used to identify situations of risk arising from this interaction, but more accurate aerodynamic analysis tools would be needed to investigate these scenarios in detail if they are perceived to be critical to the operation of the aircraft. 

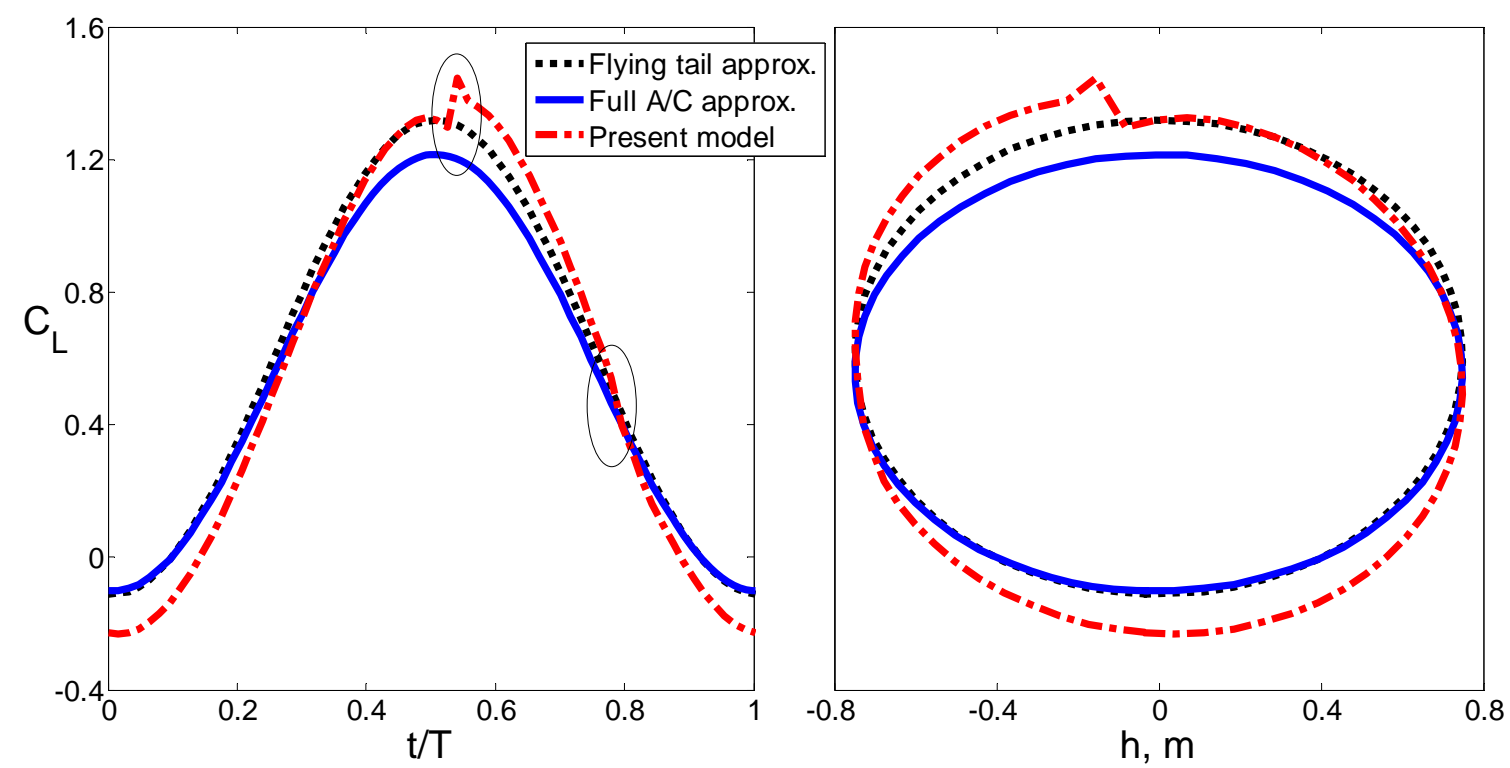

Figure 10. Tail-lift coefficient for an aircraft in plunging motion. $V_{\infty}=25 \mathrm{~m} / \mathrm{s}, \alpha_{0}=7.2 \mathrm{deg}, A_{h}=0.7487 \mathrm{~m}$, and $\omega_{h}=5 \mathrm{rad} / \mathrm{s}$.

The wake interference for the two cases is illustrated in Figure 11 and Figure 12. The lifting surfaces (main wing and tail) have been identified with darker panels. Only half of the aircraft is shown. For the first trim condition $\left(V_{\infty}=\right.$ $40 \mathrm{~m} / \mathrm{s}$ and $\alpha_{0}=2.5 \mathrm{deg}$ ), the wake never impacts on the tail - the nearest it gets is at a vertical distance of $0.62 \mathrm{~m}$ (Figure 11). In contrast, for the second condition (Figure 12, $V_{\infty}=25 \mathrm{~m} / \mathrm{s}$ and $\alpha_{0}=7.2 \mathrm{deg}$ ), the tail clearly crosses the wake at two different stations every cycle.

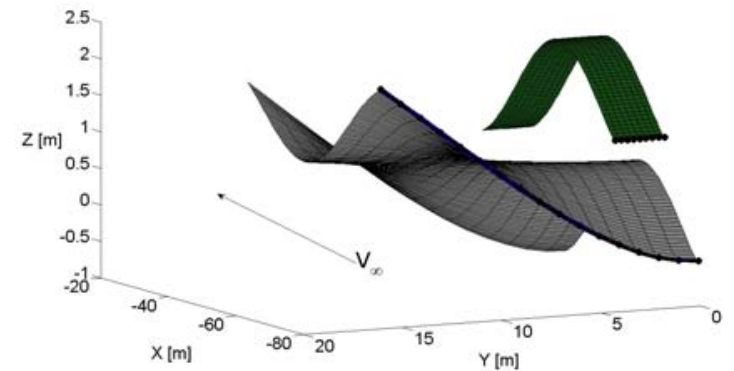

Figure 11. Wake-tail interference: no direct encounter. $V_{\infty}=40 \mathrm{~m} / \mathrm{s}, \alpha_{0}=2.5 \mathrm{deg}, A_{h}=0.7894$ $\mathrm{m}$, and $\omega_{h}=5 \mathrm{rad} / \mathrm{s}$.

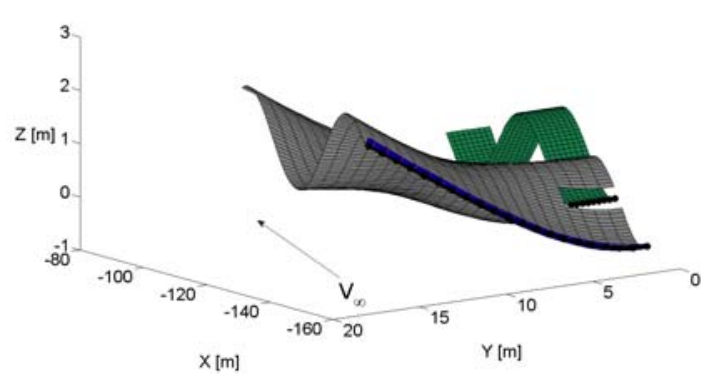

Figure 12. Wake-tail interference: tail colliding with wake. $V_{\infty}=25 \mathrm{~m} / \mathrm{s}, \alpha_{0}=7.2 \mathrm{deg}, A_{h}=0.7487$ $\mathrm{m}$, and $\omega_{h}=5 \mathrm{rad} / \mathrm{s}$.

\section{Pure pitching}

Next, a pure harmonic pitching motion (in addition to the full vehicle trim angle of attack) has been studied. In this case, the incidence angle of the aircraft does not remain constant, and it is given by

$$
\alpha=\alpha_{0}+A_{\alpha} \cos \left(\omega_{\alpha} t\right)
$$

where $\alpha_{0}$ represents the trim angle of attack, dependent on the free-stream velocity.

The pitching motion around the trim condition has been defined so that it would cancel out the induced angle of attack due to the plunging motion described above, Eq. (22). Therefore, $\omega_{\alpha}=5 \mathrm{rad} / \mathrm{s}$ and $A_{\alpha}=\tan ^{-1}\left(A_{h} \omega_{h} / V_{\infty}\right)$. Note that in this case there is no plunging motion, but the parameters of the previous sections are used to define comparable pitching motions. 
As for the plunging case, two different trim conditions have been investigated: 1) $V_{\infty}=40 \mathrm{~m} / \mathrm{s}, \alpha_{0}=2.5$ deg; and 2) $V_{\infty}=25 \mathrm{~m} / \mathrm{s}, \alpha_{0}=7.2 \mathrm{deg}$. The pitching amplitude that corresponds to these cases is, respectively: 1) $A_{\alpha}=5.6 \mathrm{deg}$, and 2) $A_{\alpha}=8.5 \mathrm{deg}$. Like in the vertical motion situation, the direct wake-tail collision only occurs for the largest trim angle. Note also that the maximum incidence angle at $V_{\infty}=25 \mathrm{~m} / \mathrm{s}$ is $\alpha=\alpha_{0}+A_{\alpha}=15.7 \mathrm{deg}$, which is probably above the stall limit - recall that the present aerodynamic model is inviscid and does not incorporate stall modeling.

Results for both cases are presented in Figure 13 and Figure 14, where the lift coefficient of the tail is plotted against time and pitching amplitude - only the "additional" dynamic pitching is taken for the plots in the right and the trim angle is subtracted from the total incidence angle.
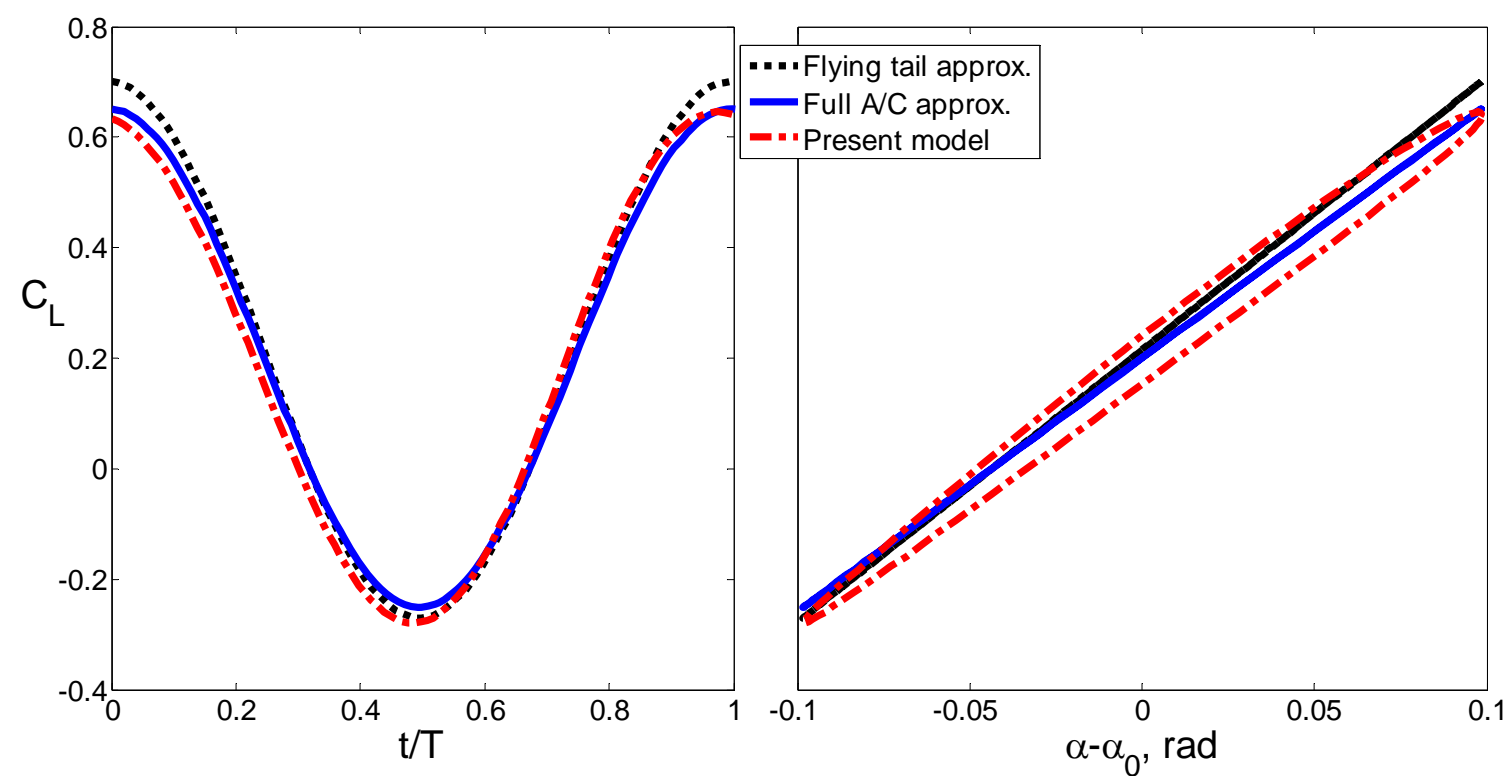

Figure 13. Tail-lift coefficient for an aircraft in pitching motion. $V_{\infty}=40 \mathrm{~m} / \mathrm{s}, \alpha_{0}=2.5 \mathrm{deg}, A_{\alpha}=5.6 \mathrm{deg}$, and $\omega_{\alpha}=5 \mathrm{rad} / \mathrm{s}$.
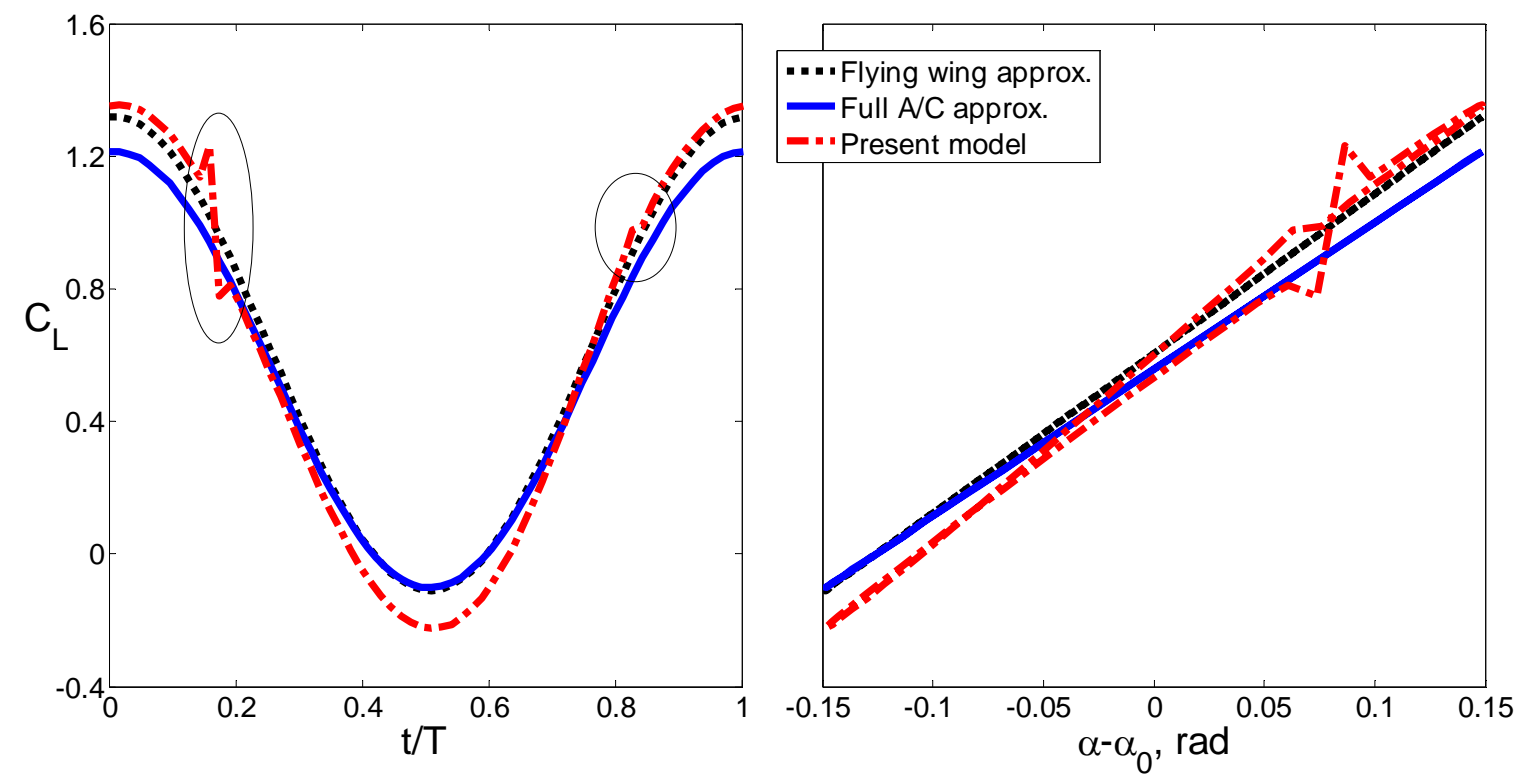

Figure 14. Tail-lift coefficient for an aircraft in pitching motion. $V_{\infty}=25 \mathrm{~m} / \mathrm{s}, \alpha_{0}=7.2 \mathrm{deg}, A_{\alpha}=8.5 \mathrm{deg}$, and $\omega_{\alpha}=5 \mathrm{rad} / \mathrm{s}$. 
The following observations can be extracted:

- The full aircraft approximation works relatively well for smaller amplitude oscillations, when no direct wakesurface encounters occur.

- However, as opposed to the purely plunging motion, the phase shift between motion and loads is not captured correctly. The hysteresis loop present in the three-dimensional results is not predicted by any of the other models. Hence, despite the fact the wake shed by the main wing does not directly collide with the tail, the interference phenomena is manifested in an alteration of the lag between motion and loads.

- When the tail cuts the wake for larger pitching amplitudes, none of the alternative methods is able to predict the resulting discontinuities. Wake-tail crossings occur for the $\left(V_{\infty}=25 \mathrm{~m} / \mathrm{s}, \alpha_{0}=7.2 \mathrm{deg}\right)$ trim condition only.

\section{Constant angle of incidence}

The last motion considered is a combination of plunging and pitching, given by

$$
\begin{gathered}
h=A_{h} \sin \left(\omega_{h} t\right) \\
\alpha=\alpha_{0}+A_{\alpha} \cos \left(\omega_{\alpha} t\right)
\end{gathered} .
$$

As in the previous cases, the frequency of oscillation has been taken to be $\omega_{\alpha}=\omega_{h}=5 \mathrm{rad} / \mathrm{s}$, very close to the $1^{\text {st }}$ bending mode of the main wing. The same trim conditions have been assumed, and therefore the plunging and pitching amplitudes coincide with the ones defined above.

As a consequence of retaining the amplitudes and frequencies from previous cases; this motion is representative of the aircraft entering a phugoid mode. This implies that the effective incidence angle remains constant at trim condition throughout the flight path: the angle of attack of the aircraft varies according to the pitching motion, but this is counteracted by the induced angle of attack due to the plunging motion.

Results for both trim conditions are plotted in Figure 15 and Figure 16. The lift coefficient of the tail is plotted against time, vertical motion, and pitching motion. These figures illustrate substantial disagreements between the present model and the alternative methods described. As in the previous analyses, the lower velocity/larger angle $\left(V_{\infty}=25 \mathrm{~m} / \mathrm{s}, \alpha_{0}=7.2 \mathrm{deg}\right)$ trim condition leads to direct tail-wake impingement, manifested by the characteristic discontinuities in the lift coefficient (Figure 16). The tail does not cut the main wing's wake for the other trim combination of parameters.
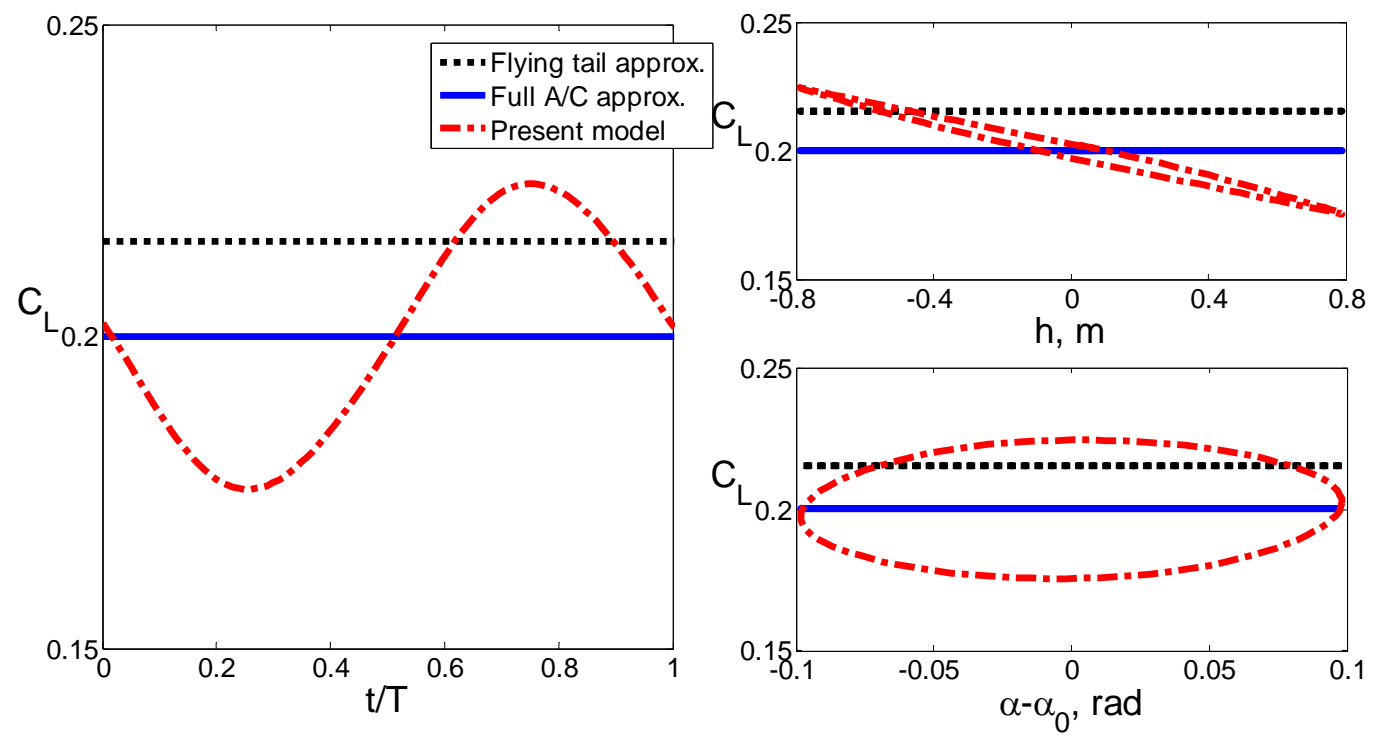

Figure 15. Tail-lift coefficient for an aircraft flying at a constant angle of incidence. $V_{\infty}=40 \mathrm{~m} / \mathrm{s}, \alpha_{0}=2.5 \mathrm{deg}$, $A_{h}=0.7894 \mathrm{~m}, A_{\alpha}=5.6 \mathrm{deg}$, and $\omega_{h}=\omega_{\alpha}=5 \mathrm{rad} / \mathrm{s}$. 

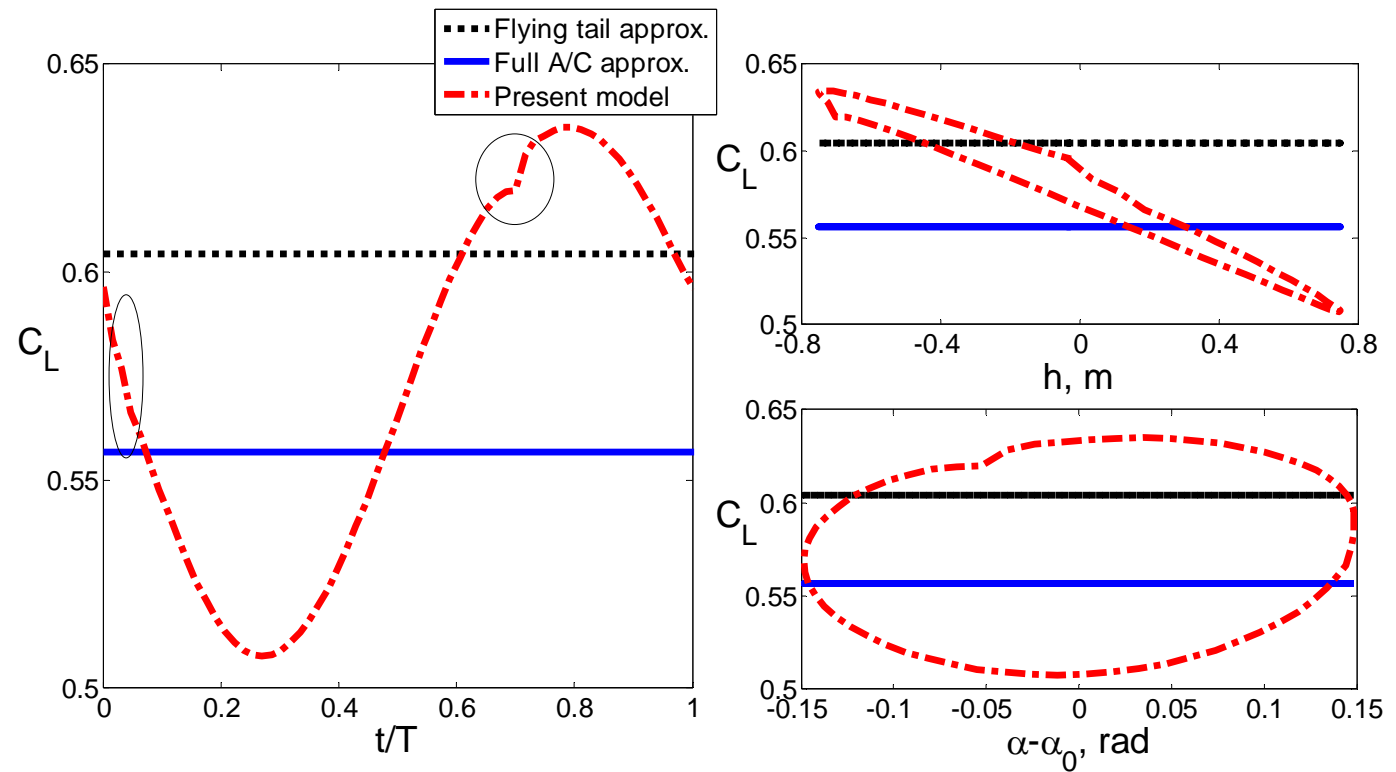

Figure 16. Tail-lift coefficient for an aircraft flying at constant angle of incidence. $V_{\infty}=25 \mathrm{~m} / \mathrm{s}, \alpha_{0}=7.2 \mathrm{deg}$, $A_{h}=0.7487 \mathrm{~m}, A_{\alpha}=8.5 \mathrm{deg}$, and $\omega_{h}=\omega_{\alpha}=5 \mathrm{rad} / \mathrm{s}$.

As the effective incidence angle remains constant throughout the flight, the proposed approximations estimate a

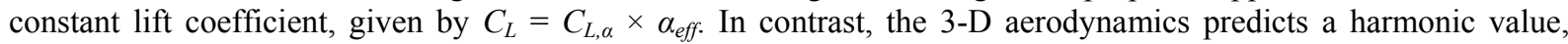
consistent with the oscillatory nature of the wake, which approaches and moves away in a cyclic fashion. Even though the amplitude of the oscillations of the lift-coefficient are not as large as in the pure plunging and pitching cases (only of the order of $10 \%$ of the average value), this phenomenon is visible for situations with and without actual crossings. Out of the three models considered, only the UVLM accounts for the evolution of the wake-shape, and thus it is the sole method that captures its influence over the tail lift coefficient.

\section{Free-wake effects}

Finally, the influence of considering free-wake effects will be investigated. As explained in Section III, the UVLM obtains the true shape of the force-free wake as part of the solution procedure. The wake is shed and convected away according to the local flow velocity, and it is thus allowed to roll up and stretch.

However, computing the rollup of the wake is a very computationally expensive process, since it requires $O\left(K_{w}^{2}\right)$ evaluations of the Biot-Savart law, Eq. (10), at each time-step $-K_{w}$ is the number of wake panels, which linearly increases with time. In addition, as the distance between the main wing and the tail is relatively large (10 m, see Figure 5) many wake panels need to be retained to account for the interference. This becomes prohibitive for a refined enough temporal and spatial discretization - for the dual-core desktop computer in which simulations have been run for this work.

For this reason, results presented so far have been obtained with the wake rollup routine disabled, i.e., the wake is shed by the trailing edges of the lifting surfaces but its shape is not modified according to the local flow velocity. This simplification is a strong one and it needs justification, since the rollup process will surely influence the lift of the tail.

The aspect-ratio of the main wing is much higher than that of the tail (6.4 times) and for the deformations considered (see Figure 8) the tips of both surfaces will be located at a great distance (over $10 \mathrm{~m}$ ). Consequently, the tip vortices released by the wing will be relatively far away from the tail, and they are therefore expected to play a minor role in the variables under study. As the wingtip approaches the tail - smaller aspect-ratio or larger static/dynamic deformations -, the influence of the wake rollup might become far from negligible.

In order to assess its impact in the cases subject to study, further simulations have been run including wake rollup. Figure 17 and Figure 18 depict the tail lift coefficient for the same model HALE aircraft experiencing harmonic plunging motions. The same two trim conditions as above have been considered, and apart from the lift coefficient computed with the wake rollup enabled, results without wake rollup and the full aircraft approximation are included. 
It can be inferred from the plots that, when no collisions occur, the rollup process has a negligible effect in the lift coefficient of the tail (Figure 17: $V_{\infty}=40 \mathrm{~m} / \mathrm{s}, \alpha_{0}=2.5 \mathrm{deg}$ ). In contrast, for trim conditions that guarantee tailwake encounters (Figure 18: $V_{\infty}=25 \mathrm{~m} / \mathrm{s}, \alpha_{0}=7.2 \mathrm{deg}$ ), the rollup does indeed influence results, slightly changing both the location and the amplitude of the discontinuities. However, the overall behavior is reasonably well captured without rollup. As the main goal of this study has been to obtain qualitative observations of the wake interference, results presented in previous sections are deemed acceptable. If the sudden changes of lift were to be accurately predicted, a rigorous convergence study would be required for temporal and spatial discretizations during the waketail impingement. Remeshing ${ }^{40}$ or discarding wake connectivity ${ }^{41,44}$ could also be considered, but this was not done here.
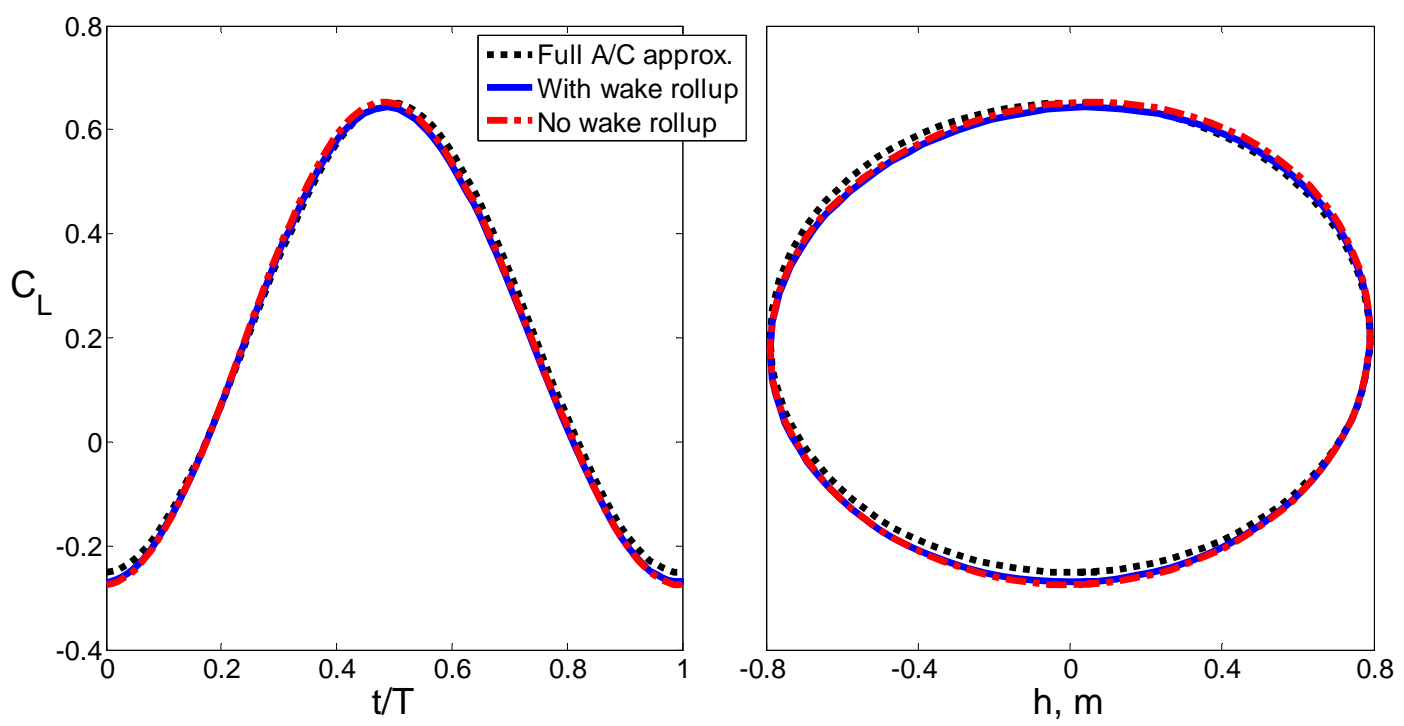

Figure 17. Influence of wake rollup on the tail of an aircraft in plunging motion. $V_{\infty}=40 \mathrm{~m} / \mathrm{s}, \alpha_{0}=2.5 \mathrm{deg}$, $A_{h}=0.7894 \mathrm{~m}$, and $\omega_{h}=5 \mathrm{rad} / \mathrm{s}$.
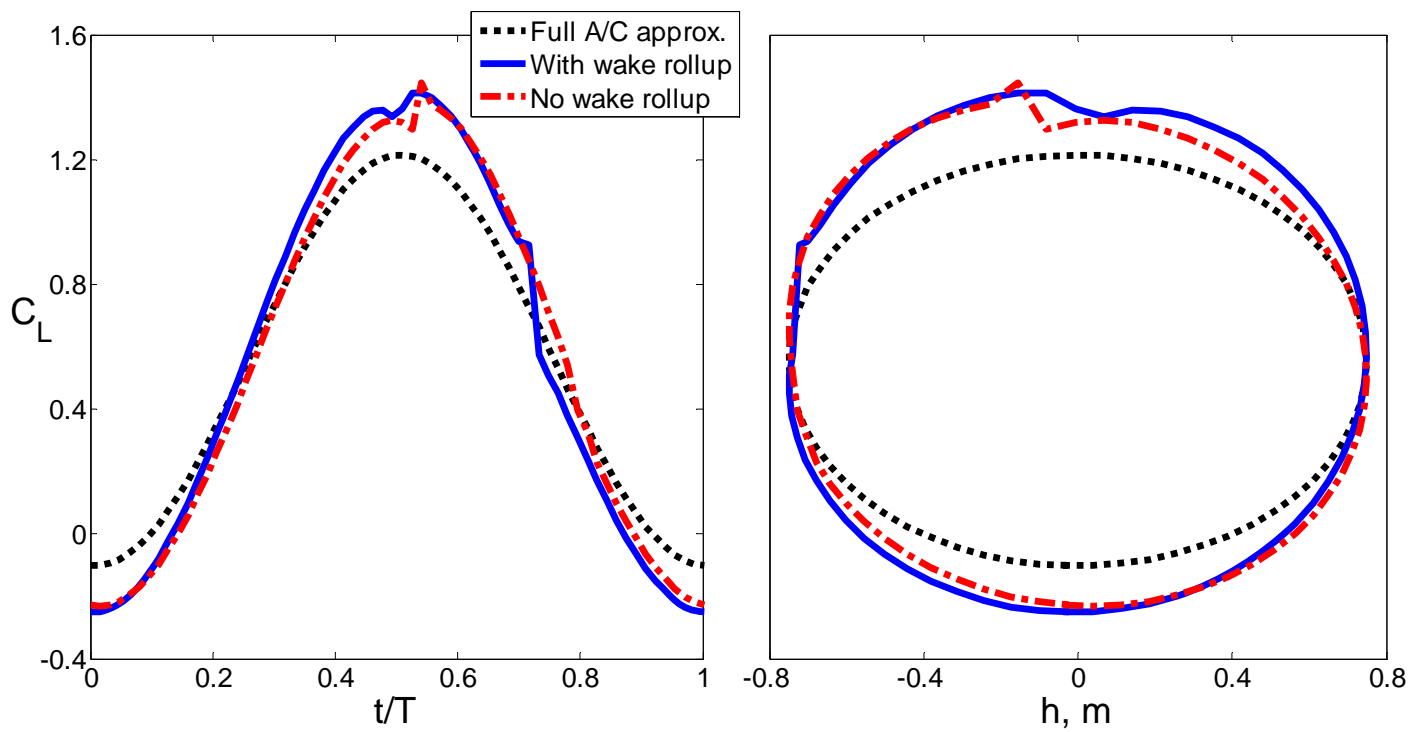

Figure 18. Influence of wake rollup on the tail of an aircraft in plunging motion. $V_{\infty}=25 \mathrm{~m} / \mathrm{s}, \alpha_{0}=7.2 \mathrm{deg}$, $A_{h}=0.7487 \mathrm{~m}$, and $\omega_{h}=5 \mathrm{rad} / \mathrm{s}$. 
The plunging, pitching and constant angle-of-incidence motions considered so far, coupled with the flexibility of the wing, lead to considerable dynamic deformations around the static trim position. Figure 19, Figure 20, and Figure 21 depict the deformed aircraft at different stations along the relevant flight-path followed during the harmonic motions described above. The trim condition corresponding to $V_{\infty}=25 \mathrm{~m} / \mathrm{s}$ and $\alpha_{0}=7.2$ deg has been considered.

The first snapshot from the right represents the beginning of a cycle: there is no vertical displacement but the pitch is maximum. At a quarter of the period, the vertical motion is at its uppermost position and the dynamic pitching is zero. The middle image depicts the aircraft halfway through the cycle. The snapshot at three-quarters of the period (fourth from the right) exhibits the lowest altitude and no extra torsion with respect to the trim angle. The final image closes the cycle.

The maximum tip deflections are $19 \%, 17 \%$ and $15 \%$ of the semi-span, for plunging, pitching and constant incidence, respectively. Note that the figures are not to scale in order to illustrate these deformations.

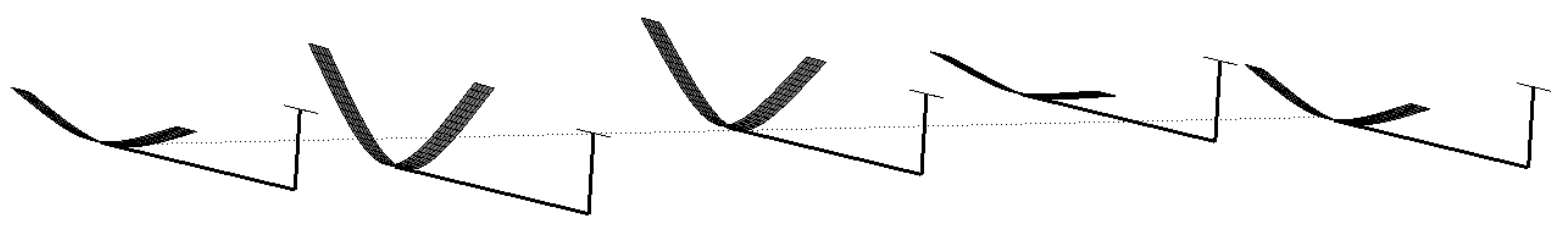

Figure 19. Deformed aircraft in plunging motion. From right to left: $t=0, t=1 / 4 T, t=1 / 2 T, t=3 / 4 \mathrm{~T}$, and $t=T$, with $T=1.26 \mathrm{~s}$. $V_{\infty}=25 \mathrm{~m} / \mathrm{s}, \alpha_{0}=7.2 \mathrm{deg}, A_{h}=0.7487 \mathrm{~m}$, and $\omega_{h}=5 \mathrm{rad} / \mathrm{s}$.

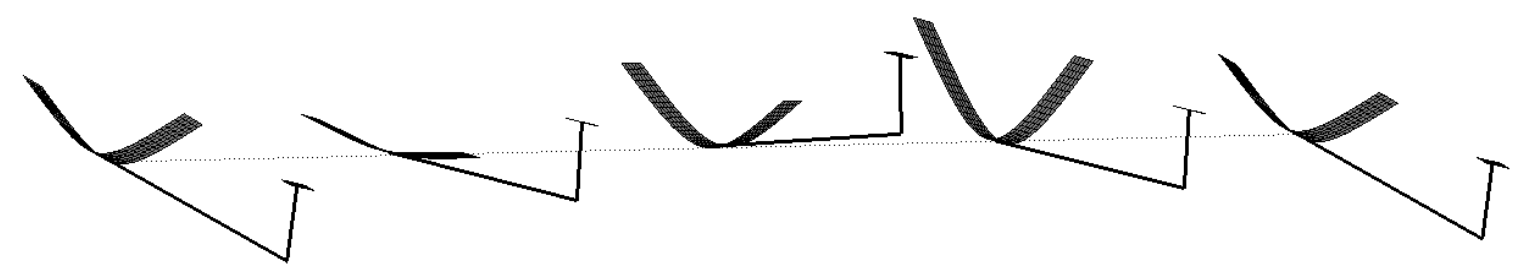

Figure 20. Deformed aircraft in pitching motion. From right to left: $t=0, t=1 / 4 T, t=1 / 2 T, t=3 / 4 \mathrm{~T}$, and $t=T$, with $T=1.26 \mathrm{~s} . V_{\infty}=25 \mathrm{~m} / \mathrm{s}, \alpha_{0}=7.2 \mathrm{deg}, A_{\alpha}=8.5 \mathrm{deg}$, and $\omega_{\alpha}=5 \mathrm{rad} / \mathrm{s}$.

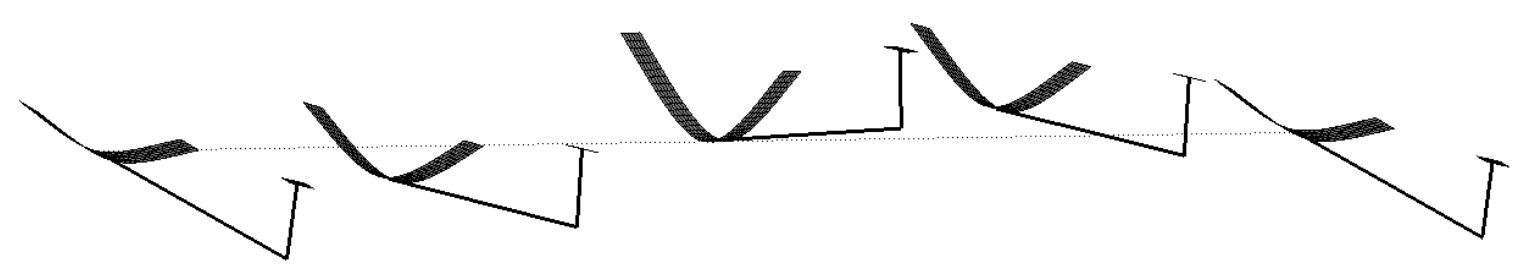

Figure 21. Deformed aircraft at constant angle of incidence. From right to left: $t=0, t=1 / 4 T, t=1 / 2 T, t=3 / 4 \mathrm{~T}$, and $t=T$, with $T=1.26 \mathrm{~s}$. $V_{\infty}=25 \mathrm{~m} / \mathrm{s}, \alpha_{0}=7.2 \mathrm{deg}, A_{h}=0.7487 \mathrm{~m}, A_{\alpha}=8.5 \mathrm{deg}$, and $\omega_{h}=\omega_{\alpha}=5 \mathrm{rad} / \mathrm{s}$. 


\section{Concluding remarks}

A unified nonlinear model has been presented for the coupled flight dynamics and aeroelasticity of very flexible aircraft. It is based on a displacement-based geometrically-exact composite beam model, discretized using finite elements, and on a general unsteady vortex lattice method, which computes the shape of the wake as part of the solution procedure (by allowing it to convect and to roll up). The combination of both the flexible-body dynamics and the aerodynamics models leads to a tightly-coupled nonlinear state-space formulation of the flexible vehicle dynamics. It offers a computationally-efficient framework for performance estimation and control synthesis of very flexible aircraft. The formulation is inherently nonlinear and easily expandable, and provides a good representation of the aircraft dynamics in situations where the local strains are small and viscous and compressibility effects are not relevant. The implementation of the structural and aerodynamic models had been partially verified in previous work against relevant results in the literature. This paper has expanded this validation exercise by showing the ability to capture the aeroelastic stability boundaries on two different configurations available in the literature (the Goland wing and a very flexible HALE aircraft concept).

The present aerodynamic model is able to capture a first approximation to the aircraft wake, and that has been used to investigate the interference between the wake of the wing and the aircraft tail for the chosen HALE configuration and for prescribed rigid-body motions of the flexible aircraft. The wake was found to have some influence on the total lift of the tail when the distance between them was of the order of the tail chord. The beamUVLM model was shown to be able to capture that interference, which is likely to occur on very flexible aircraft at low flight speeds. Furthermore, the present coupled model can also provide some relevant information when direct collisions occur between wake and tail, even though the wake is allowed to pass through the tail. In these cases, the numerical results can only be seen as indicative of the actual physical event, but can provide valuable information during the design process.

Finally, it should be noted that these numerical studies have not taken into account the flight dynamics of the aircraft. Instead, prescribed dynamics were introduced to allow a parametric evaluation of the effects of wake interference on the tail lift. The potential importance of these effects has been shown and can play a relevant role in the handling and stability characteristics of very flexible vehicles. Further work still needs to be carried out to explore the fully coupled problem, including additional convergence studies to study the effect of the temporal and spatial discretizations on the capture of the wake-tail interference.

\section{Acknowledgments}

Joseba Murua gratefully acknowledges the financial support provided by the Department of Education, Universities and Research of the Basque Government.

\section{References}

${ }^{1}$ Cesnik, C. E. S., and Su, W., "Nonlinear Aeroelastic Modeling and Analysis of Fully Flexible Aircraft," AIAA Paper No. 2005-2169, 2005.

${ }^{2}$ Noll, T. E., Brown, J. M., Perez-Davis, M. E., Ishmael, S. D., Tiffany, G. C., and Gaier, M., "Investigation of the Helios Prototype Aircraft Mishap", NASA Technical report, URL: http://www.nasa.gov/pdf/64317main helios.pdf, [cited 9 July 2010].

${ }^{3}$ Patil, M. J., Hodges, D. H., and Cesnik, C. E. S., "Nonlinear Aeroelasticity and Flight Dynamics of High-Altitude longEndurance Aircraft," Journal of Aircraft, Vol. 38 No. 1, 2001, pp. 88-94.

${ }^{4}$ Patil, M. J., Hodges, D. H., and Cesnik, C. E. S., "Limit-Cycle Oscillations of High-Aspect-Ratio Wings," Journal of Fluids and Structures, Vol. 15, No. 1, 2001, pp. 107-132.

${ }^{5}$ Romeo, G., Frulla, G., Marzoca, P., and Tuzcu, I., "Nonlinear Aeroelastic Modeling and Experiments on Flexible Wings," AIAA paper No. 2006-2186, 2006.

${ }^{6}$ Palacios, R., Cesnik, C. E. S., and Reichenbach, E. Y., "Re-examined Structural Design Procedures for Very Flexible Aircraft," 2007 International Forum of Aeroelasticity and Structural Dynamics, Stockholm, Sweden, 2007.

${ }^{7}$ Patil, M. J., and Hodges, D. H., "On the Importance of Aerodynamic and Structural Geometrical Nonlinearities in Aeroelastic Behavior of High-Aspect-Ratio Wings," Journal of Fluids and Strcutures, Vol. 19 No. 7, 2004, pp. 905-915.

${ }^{8}$ Patil, M. J., and Hodges, D. H., "Flight Dynamics of Highly Flexible Flying Wings," Journal of Aircraft, Vol. 43, No. 6, 2006, pp. 1790-1798.

${ }^{9}$ Shearer, C. M., and Cesnik, C. E. S., "Nonlinear Flight Dynamics of Very Flexible Aircraft," Journal of Aircraft, Vol. 44, No. 5, 2007, pp. 1528-1545.

${ }^{10}$ Karpel, M., "Procedures and Models for Aeroservoelastic Analysis and Design," Journal of Applied Mathematics and Mechanics,Vol. 81, No. 9, 2000, pp. 579-592. 
${ }^{11}$ Van Schoor, M. C., and von Flotow, A. H., "Aeroelastic Characteristics of a Highly Flexible Aircraft," Journal of Aircraft, Vol. 27, No. 10, 1990, pp. 901-908.

${ }^{12}$ Drela, M. (1999). "Integrated Simulation Model for Preliminary Aerodynamic, Structural, and Control-Law Design of Aircraft," AIAA Paper No. 1999-1394.

${ }^{13}$ Patil, M. J., Hodges, D. H., and Cesnik, C. E. S., "Nonlinear Aeroelastic Analysis of Complete Aircraft in Subsonic Flow," Journal of Aircraft, Vol. 37, No. 5, 2000, pp. 753-760.

${ }^{14}$ Meirovitch, L., and Tuzcu, I., "Unified Theory for the Dynamics and Control of Maneuvering Flexible Aircraft," AIAA Journal, Vol. 42, No. 4 , 2004, pp. 714-727.

${ }^{15}$ Garcia, J. A., "Numerical Investigation of Nonlinear Aeroelastic Effects on Flexible High-Aspect-Ration Wings," Journal of Aircraft, Vol. 42, No. 4 , 2005, pp. 1025-1036.

${ }^{16}$ Demasi, L., and Livne, E., "Dynamic Aeroelasticity of Structurally Nonlinear Configurations Using Linear Modally Reduced Aerodynamic Generalized Forces," AIAA Journal, Vol. 47, No. 1, 2009, pp. 71-90.

${ }^{17}$ Wang, Z., Chen, P. C., Liu, D. D., and Mook, D. T., "Nonlinear-Aerodynamics/Nonlinear-Structure Interaction Methodology for a High-Altitude Long-Endurance Wing," Journal of Aircraft, Vol. 47, No. 2, 2010, pp. 556-566.

${ }^{18}$ Palacios, R., and Cesnik, C. E. S., "Structural Models for Flight Dynamic Analysis of Very Flexible Aircraft," AIAA paper No. 2009-2403, 2009.

${ }^{19}$ Hodges, D. H., "A Mixed Variational Formulation Based on Exact Intrinsic Equations for Dynamics of Moving Beams," International Journal of Solids and Structures, Vol. 26, No. 11, 1990, pp. 1253-1273.

${ }^{20}$ Géradin, M., and Cardona, A., Flexible Multibody Dynamics. A Finite Element Approach, John Wiley and Sons, Ltd, Chichester, England, 2001.

${ }^{21}$ Simo, J. C., and Vu-Quoc, L., "A Three-Dimensional Finite- Part II: Computational Aspects," Computer Methods in Applied Mechanics and Engineering, Vol. 58, 1986, pp. 79-116.

${ }^{22}$ Cesnik, C. E. S., and Brown, E. L., "Modeling of High Aspect-ratio Active Flexible Wings for Roll Control," AIAA Paper No. 2002-1719, 2002.

${ }^{23} \mathrm{Su}$, W., and Cesnik, C. E. S., "Nonlinear Aeroelasticity of a Very Flexible Blended-Wing-Body Aircraft," AIAA Paper No. 2009-2402, 2009.

${ }^{24}$ Hodges, D. H., "Geometrically Exact, Intrinsic Theory for Dynamics of Curved and Twisted Anisotropic Beams," AIAA Journal, Vol. 41, No. 6, 2003, pp. 1131-1137.

${ }^{25}$ Peters, D. A., "Two-Dimensional Incompressible Unsteady Airfoil Theory - An Overview," Journal of Fluids and Structures, Vol. 24, No. 3, 2008, pp. 295-312.

${ }^{26}$ Leishman, J. G., and Nguyen, K. G., "State-Space Representation of Unsteady Airfoil Behavior," AIAA Journal, Vol. 28, No. 5,1989 , pp. 836-844.

${ }^{27}$ Anderson Jr, J. D., Fundamentals of Aerodynamics, McGraw-Hill, Ney York, NY, USA, 2001, Chap. 6.

${ }^{28}$ Mukherjee, R., and Gopalarathnam, A., "Post-Stall Prediction of Multiple-Lifting-Surface Configurations Using a Decambering Approach,” Journal of Aircraft, Vol. 43, No. 3, 2006, pp. 660-668.

${ }^{29}$ Laxman, V., and Venkatesan, C., "Chaotic Response of an Airfoil due to the Aeroelastic Coupling and Dynamic Stall," AIAA Journal, Vol. 45, No. 1, 2007, pp. 271-282.

${ }^{30}$ Døssing, M., "Vortex Lattice Modelling of Winglets on Wind Turbine Blades," Master's Thesis, Risø National Laboratory, Technical University of Denmark, Roskilde, Denmark, 2007.

${ }^{31}$ Rockwell, D., "Vortex-Body Interactions," Annual Review of Fluid Dynamics, Vol. 30, 1998, pp. 199-229.

${ }^{32}$ Karkehabadi, R., "Aerodynamic Interference of a Large and a Small Aircraft," Journal of Aircraft, Vol. 41, No. 6, 2004, pp. 1424-1429.

${ }^{33}$ Albano, D., and Rodden, W. P., "A Doublet-Lattice Method for Calculating Lift Distributions on Oscillating Surfaces in Subsonic Flows, AIAA Journal, Vol. 7, No. 2, 1969, pp. 279-285.

${ }^{34}$ Rodden, W. P., "The Development of the Doublet-Lattice Method," 1997 International Forum on Aeroelasticity and Structural Dynamics, Rome, Italy, 1997.

${ }^{35}$ Conlisk, A. T., "Modern Helicopter Aerodynamics," Annual Review of Fluid Dynamics, Vol. 29, 1997, pp. 515-567.

${ }^{36}$ Yao, Z. X., and Liu, D. D., "Vortex Dynamics of Blade-Blade Interaction," AIAA Journal, Vol. 36, No. 4, 1998, pp. 497504.

${ }^{37}$ Leishman, J. G., Bhagwat, M. J., and Bagai, A., "Freee-Vortex Filament Methods for the Analysis of Helicopter Rotor Wakes," Journal of Aircraft, Vol. 39, No. 5, 2002, pp. 759-775

${ }^{38}$ Tamura, A., Tsutahara, M., Kataoka, T., Aoyama, T., and Yang, C., "Numerical Simulations of Two-Dimensional BladeVortex Interactions Using Finite Difference Lattice Boltzmann," AIAA Journal, Vol. 46, No. 9, 2008, pp. 2235-2247.

${ }^{39}$ Wie, S. Y., Lee, S., and Lee, D. J., "Potential Panel and Time-Marching Free-Wake Coupling Analysis for Helicopter Rotor," Journal of Aircraft, Vol. 46, No. 3, 2009, pp. 1030-1041.

${ }^{40}$ Voutsinas, S. G., "Vortex Methods in Aeronautics: How to Make Things Work," International Journal of Computational Fluid Dynamics, Vol. 20, No. 1, 2006, pp. 3-18.

${ }^{41}$ Willis, D. J., Peraire, J., and White, J. K. "A Combined pFFT-Multipole Tree Code, Unsteady Panel Method with Vortex Particle Wakes," International Journal of Numerical Methods in Fluids, Vol. 53, 2007, pp. 1399-1422. 
${ }^{42}$ Katz, J., and Plotkin, A., Low-Speed Aerodynamics, Cambridge University Press, New York, NY, USA, 2001, Chap. 13.

${ }^{43}$ Elzebda, J. M., Mook, D. T., and Nayfeh, A. H., "Numerical Simulations of Steady and Unsteady Vorticity-Dominated Aerodynamic Interference," Journal of Aircraft, Vol. 31, No. 5, 1994, pp. 1031-1036.

${ }^{44}$ Cottet, G. H., and Koumoutsakos, P. D., Vortex Methods: Theory and Practice. Cambridge University Press, New York, NY, USA, 2000.

${ }^{45}$ Saban, D., and Whidborne, J. F., "Modeling of Wake Vortex Effects for Unmanned Air Vehicle Simulations," AIAA Paper No. 2009-5686, 2009.

${ }^{46}$ Palacios, R., and Cesnik, C. E. S., "Cross-Sectional Analysis of Non-Homogeneous Anisotropic Active Slender Structures," AIAA Journal, Vol. 43, No. 12, 2005, pp. 2624-2638.

${ }^{47}$ Phillips, W. F., Hailey, C. E., and Gebert, G. A., "A Review of Attitude Kinematics for Aircraft Flight Simulation," AIAA Paper No. 2000-4302, 2000.

${ }^{48}$ Shearer, C. M., and Cesnik, C. E. S., "Modified Generalized- $\alpha$ Method for Integrating Governing Equations of Very Flexible Aircraft," AIAA Paper No. 2006-1747, 2006.

${ }^{49}$ Fritz, T. E., and Long, L. N., "Object-Oriented Unsteady Vortex Lattice Method for Flapping Flight," Journal of Aircraft, Vol. 41, No. 6, 2004, pp. 1275-1290.

${ }^{50}$ Leishman, J. G., Principles of Helicopter Aerodynamics. Cambridge University Press, New York, NY, USA, 2006.

${ }^{51}$ Hall, K. C., "Eigenanalysis of Unsteady Flows About Airfoils, Cascades, and Wings," AIAA Journal, Vol. 32, No. 12, 1994, pp. 2426-2432.

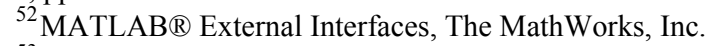

${ }^{53}$ Palacios, R., Murua, J., and Cook, R.,"Structural and Aerodynamic Models in the Nonlinear Flight Dynamics of Very Flexible Aircraft," AIAA Journal (submitted for publication), 2010.

${ }^{54}$ Goland, M., "The Flutter of a Uniform Cantilevered Wing," Journal of Applied Mechanics, Vol. 12, No. 4, 1945, pp. A197-A208.

${ }^{55}$ Wang, Z., Chen, P. C., Liu, D. D., Mook, D. T., and Patil, M. J., "Time Domain Nonlinear Aeroelastic Analysis for HALE Wings," AIAA Paper No. 2006-1640, 2006.

${ }^{56}$ Roger, K. L., “Airplane Math Modelling and Active Aeroelastic Control Design,” AGARD-CP-228, 1977, pp. 4.1-4.11.

${ }^{57}$ Wright, J. R., Cooper, J. E., Introduction to Aircraft Aeroelasticity and Loads. John Wiley \& Sons Ltd, Chichester, England, UK, 2007, pp. 412-415.

${ }^{58}$ Silva, W. A., and Bartels, R. E., "Development of Reduced-Order Models for Aeroelastic Analysis and Flutter Prediction Using the CFL3Dv6.0 Code", AIAA Paper No. 2002-1596, 2002. 